\title{
2-D Steady-State Heat Transfer Prediction in Rotating Electrical Machines Taking into account Materials Anisotropy: Thermal Resistances Network, Exact Analytical and Hybrid Methods
}

\author{
Kamel Boughrara and Frédéric Dubas
}

\begin{abstract}
This paper presents two-dimensional (2-D) thermal resistances network (TRNM), exact analytical (AM) and hybrid (HM) methods for calculating steady-state temperature and heat flux distribution in rotating electrical machines considering materials anisotropy (i.e., different thermal conductivities in both directions). They are based on the thermal equivalent circuit (TEC), the improved exact subdomain (SD) technique where the solution and thermal conductivities depend on both directions $(r, \theta)$ and the coupling between the two methods. TRNM is known as a semi-analytical method that can predict the heat transfer in the machine in less time than finite element method (Fem). The implementation of TRNM by considering the difference between the thermal conductivities in $(r, \theta)$ using its equivalence with Fem is presented. The SD technique is improved to consider the difference between thermal conductivities in the directions $(r, \theta)$. It is known that the SD technique with non-homogeneous boundary conditions (BCs) is very sensitive to the dimensions of SDs where the harmonics number and the accuracy are lower in small subdomains. Hence, the HM from the TRNM and AM is given to answer these inaccuracies especially in electrical machines with a high number of stator slots and rotor poles. The heat sources are volumetric power losses due to hysteresis, eddy-current, Joule losses and windage losses in all the regions of the machine obtained by a simplified method. The studied problem is conductive with conductive interface conditions (ICs) and convective heat transfer between the machine and the external air and at the rotor internal air. The semi-analytical results are compared between them as well as with those obtained by Fem.
\end{abstract}

Keywords-Anisotropic materials, conductive heat transfer, convection, exact subdomain technique, thermal resistances network.

\section{NOMENCLATURE}

$\begin{array}{ll}\text { TRNM } & \text { Thermal Resistances Network Method. } \\ \text { AM } & \text { Analytical Method. } \\ \text { HM } & \text { Hybrid Method. } \\ \text { TEC } & \text { Thermal Equivalent Circuit. } \\ \text { Fem } & \text { Finite element method. } \\ \text { SD } & \text { Subdomain. } \\ \text { BCs } & \text { Boundary Conditions. } \\ \text { PM } & \text { Permanent Magnet. } \\ \text { PDEs } & \text { Partial Differential Equations. } \\ \text { ICs } & \text { Interface Conditions. }\end{array}$

\section{INTRODUCTION}

Thermal modeling is used to design the insulation system of electrical machines. Currently, the Fem and TEC are the most used methods [1]-[6]. Some of them take into account materials

\footnotetext{
Manuscript received April 15, 2021; revised May 12, 2021.

This work was supported in part by the DGRSDT under Grant A01L07ES160220180002.

$K$. Boughrara is affiliated with the Electrotechnique Department, Laboratoire de Recherche en Electrotechnique (LRE), Ecole Nationale Polytechnique, Rue des Frères Oudek, Hassan Badi, B.P. 182, El-Harrach, 16200, Algiers, Algeria, (e-mail: kamel.boughrara@g.enp.edu.dz).

F. Dubas is with Energy Department, FEMTO-ST, CNRS, University of Bourgogne Franche-Comté, F90000 Belfort, France (e-mail: fdubas@univ-fcomte.fr).
}

Digital Object Identifier (DOI): 10.53907/enpesj.v1i1.25 anisotropy especially in the $z$-direction with a threedimensional (3-D) study. Recently, Boughrara et al. (2018) [7] introduced a new 2-D exact SD technique able to predict steady-state heat transfer in rotating electrical machines without considering the anisotropy of thermal conductivities. This model is based on the Dubas' superposition technique [8]-[9] developed for the prediction of the magnetic field in air- or ironcored coil. This method is very accurate and can be used for different topologies of synchronous and asynchronous machines.

The thermal modeling of electrical machines using TEC in steady-state and/or transient is fast with acceptable accuracy compared to Fem and AM, especially when the number of nodes and thermal resistances is low. TRNM is more accurate than TEC with higher time consumption [10]-[11]. However, in reality, it is not widely used for thermal design. The numerical thermal model is used in a second stage of the design to verify the temperature distribution given by semi-analytical methods. In various methods, the power losses are used as heat sources or coupled directly to electromagnetic analysis. Fem is also used with computational fluid dynamics to model convective problems inside the electrical machine and the different type of cooling. Recently, there are a few references that used an HM. TRNM of the stator and rotor are coupled to an exact AM only in the air-gap [10]-[11].

In this paper, we present three semi-analytical methods, viz., i) TRNM, ii) AM and iii) HM. TRNM is considered as Fem where the steps of meshing, materials definition with different thermal conductivities in the directions $(r, \theta)$, elementary matrix/vector and introduction of BCs exist. The model presented in [7], based on the exact SD technique, is improved to consider the materials anisotropy in the directions $(r, \theta)$ for the prediction of 
steady-state temperature and heat flux in rotating electrical machines. AM is suitable for the SDs with high dimensions where the harmonics number can be high to achieve very good accuracy.

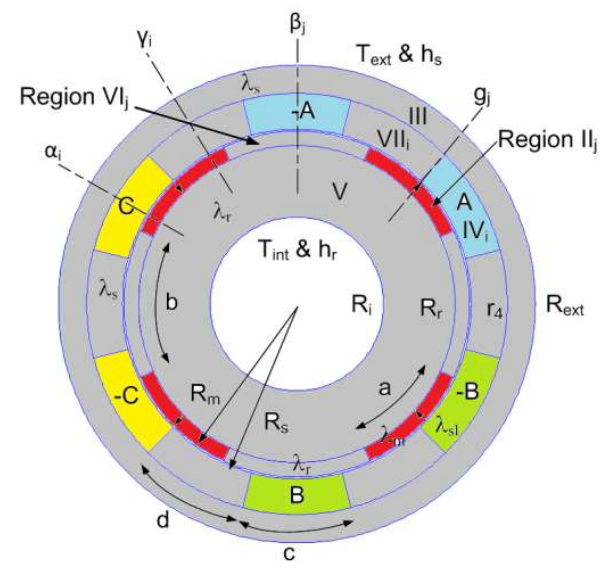

Fig. 1: Studied inset-PM machine [7].

This is the case of electrical machines with a low number of stator slots and rotor poles. In electrical machines with a high number of poles and stator slots, it is interesting to use the HM (i.e., the coupling between TRNM and AM) to simplify the thermal model and improve the accuracy. The used HM also considers the materials anisotropy. TRNM is used to model the stator slots and teeth SDs and rotor permanent-magnets (PMs) and teeth SDs while the AM is used to model the stator, rotor and air-gap. The coupling between TRNM and AM is achieved by using the discrete Fourier series at the ICs to satisfy the temperature continuity [12].

The developed semi-analytical methods are used to determine the heat transfer in inset-PM machines. Although, it is valid for most rotating electrical machines [7].

To determine the heat sources, a simple method is used in this paper to determine the power losses for the studied inset-PM machine [13]. Although, AM and magnetic resistances network method (MRNM) can be used [14]-[16]. The semi-analytical results are compared between them and with those obtained by Fem [17].

\section{TEMPERATURE CALCULATION METHODS}

The steady-state heat transfer studied in this paper is conductive with volumetric power sources and convective at the ambient air and the rotor shaft. The model is adopted with the following assumptions:

- $\quad$ The materials are considered anisotropic having different constant thermal conductivities in both directions without any variation with temperature;

- The stator and rotor slots have radial sides;

- The heat sources are volumetric, uniform and constant in each SD;

- The radiation outside stator and inside the rotor is ignored;

- The interfaces between all regions are considered perfect without any contact resistance.

The analyzed inset-PM machine has 6-slots/4-poles [see Fig. 1].

\section{A. Thermal Resistance Network Method (TRNM)}

The studied inset-PM machine is modeled using TRNM with the following steps:
The step of meshing is performed in the same way as in Fem with the use of circular elements having a node in the middle of each element. First, we define $m s+1$ radii $w r s(i)$ with $i$ vary from 1 to $m s+1$ and $n s+1$ angles $w t(j)$ with $j$ vary from 1 to $n s+1$. The number of nodes which is equal to the number of elements is $m s \times n 1$. The numbering and coordinates of nodes are given by

For i from 1 to $\mathrm{ms}$ do

For j from 1 to $n s$ do

$\operatorname{nods}(i, j)=j+(i-1) \cdot n s$;

$l=\operatorname{nods}(i, j)$;

$x(l)=\frac{w r s(i+1)+w r s(i)}{2} \cos \left(\frac{w t(j+1)+w t(j)}{2}\right) ;$

$y(l)=\frac{w r s(i+1)+w r s(i)}{2} \sin \left(\frac{w t(j+1)+w t(j)}{2}\right) ;$

end do

end do

In Fig. 2, we can show an example of mesh with 32 elements and nodes (i.e., $m s=4$ and $n s=8$ ). In this example, each element has a thickness of $w r s(i+1)-w r s(i)$ and opening of $w t(j+1)-w t(j)$. This example is introduced for clarity in the implementation of TRNM:

$$
\begin{aligned}
& w r s=10^{-3} \cdot\left[\begin{array}{lllll}
30 & 60 & 90 & 120 & 150
\end{array}\right]
\end{aligned}
$$

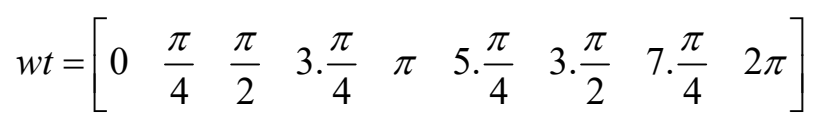

As it can be shown in (1), the numbering is done starting with 1 in the $\theta$-direction than after in the $r$-direction. From the two first radii, we have 8 elements and 8 nodes numbered from 1 to 8 from right to left. The next two radii are numbered from 9 to 16 and the same for the other radii.

\section{2- Connectivity Matrix}

From Figs. $2 \sim 4$, we can show that the node 9 is connected to 4 nodes $(10,1,16,17)$. This is the case for the entire mesh with elements having 4 thermal resistances. Supplementary nodes are added to the first and last radii to represent the $\mathrm{BC}$ between the stator and the external air and the rotor with the shaft. The connection between the nodes permits to obtain a matrix called connectivity matrix $i s k s(5, k)$ as in Fem. For internal elements that are not situated in the boundaries, we define the matrix isks as

For $i$ from 2 to $m s-1 d o$

For $j$ from 2 to $n s-1$ do

$k=\operatorname{nods}(i, j)$;

$i s k s(1, k)=\operatorname{nods}(i, j-1)$;

$i s k s(2, k)=\operatorname{nods}(i, j)$;

$i s k s(3, k)=\operatorname{nods}(i, j+1)$;

$i s k s(4, k)=\operatorname{nod} s(i-1, j)$;

$i \operatorname{sks}(5, k)=\operatorname{nods}(i+1, j)$; 


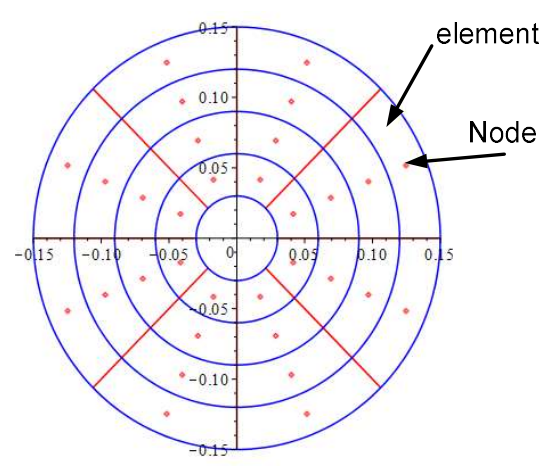

Fig. 2: Example of meshing with circular elements.

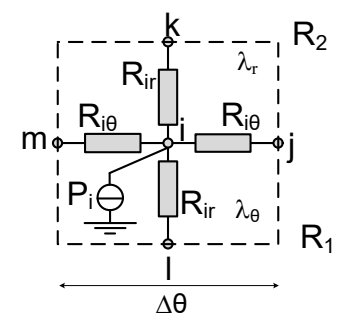

(a)

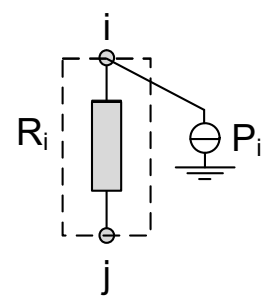

(b)
Fig. 3: Thermal resistances of an element.

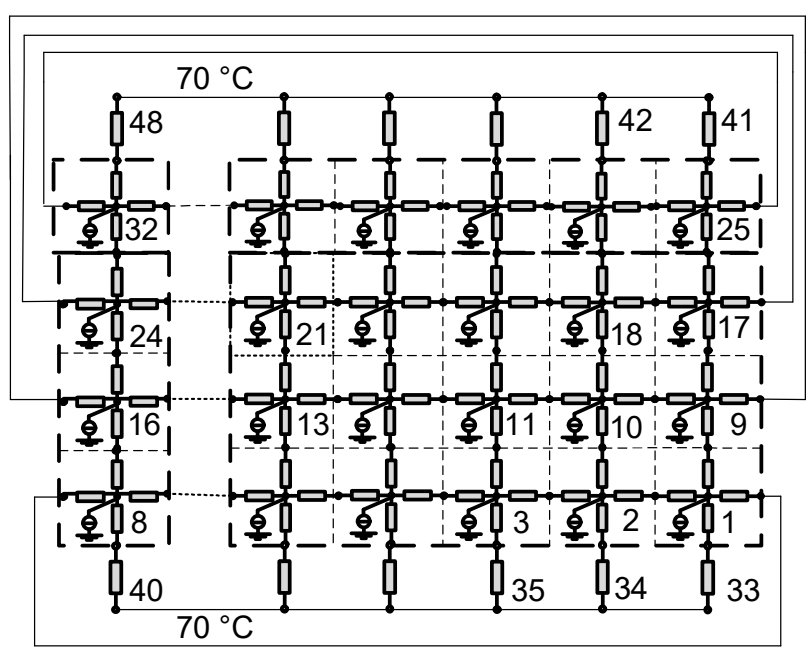

Fig. 4: TRNM representation of the example mesh.

Boundary elements have some nodes without connectivity and the connectivity value is zero. Generally, two types of elements can be used in TRNM and can have 1, 2, 3, 4 or 5 nodes [see Figs. 3(a) and (b)]. The elements can have 4, 3, 2 or 1 thermal resistance.

The thermal resistance for elements with 1 resistance can be radial or tangential. However, for elements with 4 thermal resistances, we have 2 radial and 2 tangential resistances. The element shown in Fig. 3 is used to represent thermal conduction [see Fig. 3(a)] and convection [see Fig. 3(b)] respectively. In this paper, the internal elements with a node in the center and 4 thermal resistances are used to represent the thermal conduction by

$$
R_{i r}=\frac{1}{2} \frac{\ln \left(\frac{R_{2}}{R_{1}}\right)}{\lambda_{r} \Delta \theta L_{u}}=\frac{1}{2} \frac{\ln \left(\frac{w r s(i+1)}{w r s(i)}\right)}{\lambda_{r}(w t(j+1)-w t(j)) L_{u}}
$$

$$
R_{i \theta}=\frac{1}{2} \frac{\Delta \theta}{\lambda_{\theta} L_{u} \ln \left(\frac{R_{2}}{R_{1}}\right)}=\frac{1}{2} \frac{w t(j+1)-w t(j)}{\lambda_{\theta} L_{u} \ln \left(\frac{w r s(i+1)}{w r s(i)}\right)}
$$

where $\lambda_{r}$ and $\lambda_{\theta}$ are the thermal conductivity in the $r$ - and $\theta$ direction; $R_{1}, R_{2}$ and $\Delta \theta$ are respectively the internal, the external radii and the opening of element; and $L_{u}$ the axial length of the machine.

The conductivity in anisotropic material is a tensor with

$$
[\lambda]=\left[\begin{array}{cc}
\lambda_{r} & 0 \\
0 & \lambda_{\theta}
\end{array}\right]
$$

It is interesting to note that the conductivities $\lambda_{r \theta}$ and $\lambda_{\theta r}$ are considered null for the studied machine. In polar coordinates, the thermal conductivity tensor $[\lambda]$ can be obtained from the thermal conductivity tensor in Cartesian coordinates as [18]

$$
\begin{aligned}
{\left[\begin{array}{ll}
\lambda_{r r} & \lambda_{r \theta} \\
\lambda_{r \theta} & \lambda_{\theta \theta}
\end{array}\right]=} & {\left[\begin{array}{cc}
\cos \theta & \sin \theta \\
-\sin \theta & \cos \theta
\end{array}\right]\left[\begin{array}{cc}
\lambda_{x x} & \lambda_{x y} \\
\lambda_{x y} & \lambda_{y y}
\end{array}\right] } \\
& \cdot\left[\begin{array}{cc}
\cos \theta & -\sin \theta \\
\sin \theta & \cos \theta
\end{array}\right]
\end{aligned}
$$

It is important to note that Fem uses the Cartesian coordinate's tensor for the thermal conductivity [17] and [19]. For this, the validation of the results considering the materials anisotropy in polar coordinates is done with TRNM and AM.

The boundary elements [see Fig. 4] with 1 node at fixed temperature (viz., $70^{\circ} \mathrm{C}$ ) represent the thermal convection resistance as follows

$$
\begin{gathered}
R_{r k}=\frac{1}{h_{s} L_{u}(w t(j+1)-w t(j)) w r s(m s+1)} \\
R_{r k}=\frac{1}{h_{r} L_{u}(w t(j+1)-w t(j)) w r s(1)}
\end{gathered}
$$

where $h_{s}$ and $h_{r}$ are respectively the convection coefficients at the external radius and at the shaft of the machine.

In the proposed method, the Dirichlet's condition (without convection) can be introduced by setting the thermal resistance of convection to zero.

For the mesh example in Fig. 4, there are 48 equations corresponding to 48 elements and nodes. Each conductive element has an internal volumetric source of heat $P_{i}$ and connected to four nodes. All elements can be represented similarly to equations of elements 1 and 2 by

$$
\begin{array}{r}
P_{1}=\frac{T_{1}-T_{8}}{R_{1 \theta}+R_{8 \theta}}+\frac{T_{1}-T_{2}}{R_{1 \theta}+R_{2 \theta}}+\frac{T_{1}-T_{33}}{R_{1 r}+R_{33}}+\frac{T_{1}-T_{9}}{R_{1 r}+R_{9 r}} \\
P_{2}=\frac{T_{2}-T_{1}}{R_{2 \theta}+R_{1 \theta}}+\frac{T_{2}-T_{3}}{R_{2 \theta}+R_{3 \theta}}+\frac{T_{2}-T_{34}}{R_{2 r}+R_{34}}+\frac{T_{2}-T_{10}}{R_{2 r}+R_{10 r}}
\end{array}
$$


The 16 equations of convection at boundaries are similar to those of elements 41 and 34, e.g.,

$$
\begin{gathered}
0=\frac{T_{41}-T_{25}}{R_{25 r}+R_{41}} \\
0=\frac{T_{34}-T_{2}}{R_{2 r}+R_{34}}
\end{gathered}
$$

where $T_{41}=T_{34}=70^{\circ} \mathrm{C}$.

In the case of Dirichlet's conditions, the thermal resistances of convection are fixed at infinity (i.e., $R_{41}=R_{34}=\infty$ ). A fixed heat flux BC can be considered. In this case, (16) and (17) can be modified as follows

$$
\begin{gathered}
P_{41}=\frac{T_{41}-T_{25}}{R_{25 r}} \\
P_{34}=\frac{T_{34}-T_{2}}{R_{2 r}}
\end{gathered}
$$

where $P_{34}$ and $P_{41}$ are imposed heat fluxes. The periodicity condition is satisfied by connecting nodes 1 and 8,9 and 16, 17 and 24,25 and 32 .

\section{3- Global Matrix}

The 48 equations are represented in matrix form $\operatorname{gm}(48,48)$ without replacing the known temperatures at the BCs. The second member of the system represents the entire values of $P_{i}$ with a vector $f(48)$ which represent the power losses in the machine. We begin the assembly gm with the internal elements of the conductive problem, then the equations of thermal convection at the rotor shaft and the stator ambient air. The introduction of $\mathrm{BCs}$ by convection is given by the thermal resistances and the fixed temperature (viz., $70^{\circ} \mathrm{C}$ ) at the rotor shaft and the external air of the machine. The fixed temperatures are introduced into the global matrix and vector using high diagonal numbers in the global matrix and vector. These steps for constructing the global matrix are given in Appendix A.

The global matrix and vector for the 48 unknown temperatures are solved by direct method.

$$
T=g m^{-1} \cdot f
$$

The obtained solution vector $T(48)$ allows us to calculate the density of heat flux. The radial density of heat flux for each element can be calculated by

$$
\begin{aligned}
& q(i s k s(4, k), k)=\frac{T_{k}-T_{i s k s(4, k)}}{S_{k r}\left(R_{k r}+R_{i s k s(4, k) r}\right)} \\
& q(i \operatorname{sks}(5, k), k)=\frac{T_{k}-T_{i s k s(5, k)}}{S_{k r}\left(R_{k r}+R_{i s k s(5, k) r}\right)}
\end{aligned}
$$

$$
\begin{aligned}
& q(i \operatorname{sis}(1, k), k)=\frac{T_{k}-T_{i s k s(1, k)}}{S_{k \theta}\left(R_{k \theta}+R_{i s k s(1, k) \theta}\right)} \\
& q(i \operatorname{sis}(3, k), k)=\frac{T_{k}-T_{i s k s(3, k)}}{S_{k \theta}\left(R_{k \theta}+R_{i s k s(3, k) \theta}\right)}
\end{aligned}
$$

where $S_{k r}$ and $S_{k \theta}$ are respectively the surface of an element in the $r$ - and $\theta$-direction.

For the analyzed inset-PM machine with TRNM, $m s=36$, $n s=360$, the total number of elements and nodes is 12,960 . The number of additional nodes to consider convection heat transfer at the external radius is 360 and at the shaft is 360 . The dimensions of the global matrix and vector are respectively $13,680 \times 13,680$ and 13,680 .

\section{B. Analytical Method (AM)}

\section{1- Problem Description, Assumptions and Partial Differential Equations (PDEs)}

In this section, we have improved the AM developed in [7] to consider the materials anisotropy in both directions $(r, \theta)$. The machine is subdivided into 7 regions, viz., Region I for the airgap, Region IIj for the PMs, Region III for the stator yoke, Region IVi for the stator slots, Region V for the rotor yoke, Region VIj for the rotor teeth, and Region VIIi for the stator teeth.

In steady-state, PDEs representing the temperature distribution in each region are given by

- $\quad$ in Region I by

$$
\frac{\lambda_{e r}}{r} \frac{\partial}{\partial r} T I+\lambda_{e r} \frac{\partial^{2}}{\partial r^{2}} T I+\frac{\lambda_{e \theta}}{r^{2}} \frac{\partial^{2}}{\partial \theta^{2}} T I=-p_{e}
$$

where $\lambda_{e r}$ and $\lambda_{e \theta}$ are respectively the thermal conductivities (in $W / m K$ ) of the air-gap in the $r$-and $\theta$-direction, and $p_{e}$ is the windage loss density (in $W / \mathrm{m}^{3}$ ).

- $\quad$ in Regions IIj by

$$
\frac{\lambda_{m r}}{r} \frac{\partial}{\partial r} T I I_{j}+\lambda_{m r} \frac{\partial^{2}}{\partial r^{2}} T I I_{j}+\frac{\lambda_{m \theta}}{r^{2}} \frac{\partial^{2}}{\partial \theta^{2}} T I I_{j}=-P m_{j}
$$

where $\lambda_{m r}$ and $\lambda_{m \theta}$ are respectively the thermal conductivities (in $W / m K$ ) of PMs in the $r$ - and $\theta$-direction, and $P m_{j}$ the power loss density of the $j^{\text {th }}$ Region II (in $W / m^{3}$ ).

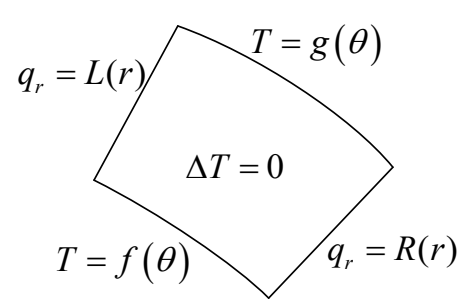

(a) Non-homogenous BCs. 


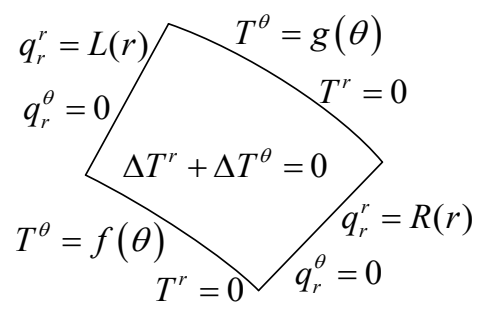

(b) Principle of superposition.

Fig. 5: Region with non-homogenous BCs [7].

- $\quad$ in Region III by

$$
\frac{\lambda_{s r}}{r} \frac{\partial}{\partial r} T I I I+\lambda_{s r} \frac{\partial^{2}}{\partial r^{2}} T I I I+\frac{\lambda_{s \theta}}{r^{2}} \frac{\partial^{2}}{\partial \theta^{2}} T I I I=-p_{s}
$$

where $\lambda_{s r}$ and $\lambda_{s \theta}$ are respectively the thermal conductivities (in $W / m K$ ) of the stator yoke in the $r$ - and $\theta$-direction, and $p_{s}$ the power loss density in the stator iron (in $W / \mathrm{m}^{3}$ ). This power loss is considered uniform and constant in the whole stator iron.

- $\quad$ in Regions IVi by

$$
\frac{\lambda_{s l t r}}{r} \frac{\partial}{\partial r} T I V_{i}+\lambda_{s l t r} \frac{\partial^{2}}{\partial r^{2}} T I V_{i}+\frac{\lambda_{s l t \theta}}{r^{2}} \frac{\partial^{2}}{\partial \theta^{2}} T I V_{i}=-P s l_{i}
$$

where $\lambda_{\text {sltr }}$ and $\lambda_{\text {slt } \theta}$ are respectively the thermal conductivities (in $W / m K$ ) of stator slot in the $r$ - and $\theta$-direction, and $P s l_{i}$ the Joule and proximity losses densities (in $\mathrm{W} / \mathrm{m}^{3}$ ). This power is considered uniform and constant in each stator slot.

- $\quad$ in Region V by

$$
\frac{\lambda_{r r}}{r} \frac{\partial}{\partial r} T V+\lambda_{r r} \frac{\partial^{2}}{\partial r^{2}} T V+\frac{\lambda_{r \theta}}{r^{2}} \frac{\partial^{2}}{\partial \theta^{2}} T V=-p_{r}
$$

where $\lambda_{r r}$ and $\lambda_{r \theta}$ are respectively the thermal conductivities (in $W / m K$ ) of rotor yoke in the $r$ - and $\theta$-direction, and $p_{r}$ the power loss density in the rotor iron (in $\mathrm{W} / \mathrm{m}^{3}$ ). This power loss is considered uniform and constant in the rotor iron.

- $\quad$ in Regions VIj by

$$
\frac{\lambda_{a r}}{r} \frac{\partial}{\partial r} T V I_{j}+\lambda_{a r} \frac{\partial^{2}}{\partial r^{2}} T V I_{j}+\frac{\lambda_{a \theta}}{r^{2}} \frac{\partial^{2}}{\partial \theta^{2}} T V I_{j}=-P d r_{j}
$$

where $\lambda_{a r}$ and $\lambda_{a \theta}$ are respectively the thermal conductivities (in $W / m K$ ) of rotor tooth in the $r$ - and $\theta$-direction, and $P d r_{j}$ the power loss density in the rotor tooth (in $\mathrm{W} / \mathrm{m}^{3}$ ). This power loss is considered uniform and constant in the rotor tooth.

- $\quad$ in Regions VIIi by

$$
\frac{\lambda_{d s r}}{r} \frac{\partial}{\partial r} T V I I_{i}+\lambda_{d s r} \frac{\partial^{2}}{\partial r^{2}} T V I I_{i}+\frac{\lambda_{d s \theta}}{r^{2}} \frac{\partial^{2}}{\partial \theta^{2}} T V I I_{i}=-P d s_{i}
$$

where $\lambda_{d s r}$ and $\lambda_{d s \theta}$ are respectively the thermal conductivities (in $W / m K$ ) of the stator tooth in the $r$-and $\theta$-direction, and $P d s_{i}$ the power loss density in the stator tooth (in $\mathrm{W} / \mathrm{m}^{3}$ ). This power loss is considered uniform and constant in the stator tooth.
Using $\boldsymbol{q}=-[\lambda] \cdot \nabla \boldsymbol{T}$, the heat flux density components (in $\mathrm{W} / \mathrm{m}^{2}$ ) in polar coordinates are defined as

$$
\begin{aligned}
& q_{r}=-\lambda_{r} \frac{\partial T(r, \theta)}{\partial r} \\
& q_{\theta}=-\frac{\lambda_{\theta}}{r} \frac{\partial T(r, \theta)}{\partial \theta}
\end{aligned}
$$

where $\lambda_{r}$ and $\lambda_{\theta}$ are respectively the thermal conductivities in the $r$ - and $\theta$-direction.

\section{2- Temperature Solution in each $S D$}

The steady-state heat transfer in the inset-PM machine is studied using the improved 2-D exact SD technique presented in [7]. The general solutions of the above PDEs in nonhomogenous BCs [see Fig. 5(a)] are deduced by applying the superposition principle [8]-[9] [see Fig. 5(b)] and using the Fourier's series as well as the separation of variables method. The Laplace's equations in Region I, III and $\mathrm{V}$ have homogeneous BCs and Region II, IV, VI and VII present nonhomogeneous BCs. The solution of Laplace's equation

$$
\frac{\lambda_{r}}{r} \frac{\partial}{\partial r} T(r, \theta)+\lambda_{r} \frac{\partial^{2}}{\partial r^{2}} T(r, \theta)+\frac{\lambda_{\theta}}{r^{2}} \frac{\partial^{2}}{\partial \theta^{2}} T(r, \theta)=0
$$

Using the separation of variables method by replacing $T(r, \theta)=R(r) \Theta(r, \theta)$ gives

$$
\begin{gathered}
\frac{r \lambda_{r} \frac{d R(r)}{d r}}{R(r)}+\frac{r^{2} \lambda_{r} \frac{d^{2} R(r)}{d r^{2}}}{R(r)}=\mu^{2} \text { and } / \text { or }-\mu^{2} \\
-\frac{\lambda_{\theta} \frac{d^{2} \Theta(\theta)}{d \theta^{2}}}{\Theta(\theta)}=\mu^{2} \text { and } / \text { or }-\lambda^{2}
\end{gathered}
$$

where $\mu^{2}$ is positive constant.

For a positive constant equal to $\mu^{2}$, the solutions are

$$
\begin{gathered}
R_{1}(r)=C 1 \cdot r^{\frac{\mu}{\sqrt{\lambda_{r}}}}+C 2 \cdot r^{-\frac{\mu}{\sqrt{\lambda_{r}}}} \\
\Theta_{1}(\theta)=C 3 \sin \left(\frac{\mu \theta}{\sqrt{\lambda_{\theta}}}\right)+C 4 \cos \left(\frac{\mu \theta}{\sqrt{\lambda_{\theta}}}\right)
\end{gathered}
$$

For a negative constant equal to $-\mu^{2}$, the solutions are

$$
\begin{aligned}
& R_{2}(r)=E 1 \cdot \cos \left(\frac{\mu \ln (r)}{\sqrt{\lambda_{r}}}\right)+E 2 \cdot \sin \left(\frac{\mu \ln (r)}{\sqrt{\lambda_{r}}}\right) \\
& \Theta_{2}(\theta)=E 3 \sinh \left(\frac{\mu \theta}{\sqrt{\lambda_{\theta}}}\right)+E 4 \cosh \left(\frac{\mu \theta}{\sqrt{\lambda_{\theta}}}\right)
\end{aligned}
$$

For the constant equal to zero, the solutions are

$$
R_{3}(r)=B 1+B 2 \ln (r)
$$




$$
\Theta_{3}(\theta)=A 1 . \theta+A 2
$$

In Region I, III and V with the homogenous BCs and periodicity equal to $2 \pi$, the constant $\mathrm{A} 1=0$ and $\mu=n \sqrt{\lambda_{\theta}}$ (with $n$ is a positive integer). The periodic regions II, IV, VI and VII in the $r$ and $\theta$-direction with the non-homogeneous BCs have the constant $\mathrm{A} 1=0$ and $\mu=\frac{n \pi}{a} \sqrt{\lambda_{\theta}}$ in the $\theta$-direction for PMs region where $a$ is the PM-opening angle and $\mu=\frac{n \pi}{\ln \left(\frac{r_{2}}{r_{1}}\right)} \sqrt{\lambda_{r}}$ in the $r$-direction. The particular solution of Poisson's equations (28) to (34) in each SD is given by

$$
T_{p}=-p r^{2} / 4 \lambda_{r}
$$

where $p$ is a volumetric constant power loss in each SD.

The solution of (28) in the air-gap with the homogenous BCs is given for each harmonic $n$ by

$$
T I(r, \theta)=\mid \begin{aligned}
& R_{3}(r) \Theta_{3}(r, \theta)+R_{1}(r) \Theta_{1}(r, \theta) \\
& \cdots+T_{p}
\end{aligned}
$$

and can be written as

$$
\begin{aligned}
& T I(r, \theta)=-p r^{2} /\left(4 \lambda_{e r}\right)+A 1_{0}+A 2_{0} \ln (r) \\
& \mathrm{L}+\sum_{n=1}^{n n}\left(A 1_{n}\left(\frac{r}{R_{s}}\right)^{n . \tau_{e}}+A 2_{n}\left(\frac{r}{R_{m}}\right)^{-n \cdot \tau_{e}}\right) \sin (n \theta) \\
& \mathrm{L}+\sum_{n=1}^{n n}\left(A 3_{n}\left(\frac{r}{R_{s}}\right)^{n \cdot \tau_{e}}+A 4_{n}\left(\frac{r}{R_{m}}\right)^{-n \cdot \tau_{e}}\right) \cos (n \theta)
\end{aligned}
$$

where $\tau_{e}=\sqrt{\lambda_{e \theta} / \lambda_{e r}}$, and $p_{e}$ the windage loss in the air-gap.

The solution of (29) in Region IIj with the non-homogenous $\mathrm{BCs}$ is given for each harmonic by

$$
T I I_{j}=\mid \begin{aligned}
& R_{3}(r) \Theta_{3}(r, \theta)+R_{1}(r) \Theta_{1}(r, \theta)+R_{2}(r) \Theta_{2}(r, \theta) \\
& \cdots+T_{p}
\end{aligned}
$$

and can be reduced using BCs presented in Fig. 5 as

$$
\begin{aligned}
& \text { TII }(r, \theta)=B 1_{j, 0}+B 2_{j, 0} \ln (r)-P m_{j} r^{2} /\left(4 \lambda_{m r}\right) \\
& +\sum_{m=1}^{m m}\left(B 1_{j, m}\left(\frac{r}{R_{m}}\right)^{f r a_{m} \cdot \tau_{m}}+B 2_{j, m}\left(\frac{r}{R_{r}}\right)^{-f r a_{m} \cdot \tau_{m}}\right) \cos \left(f r a_{m}\left(\theta-\theta 1_{j}\right)\right) \\
& +\sum_{k=1}^{k k}\left(\begin{array}{c}
\operatorname{sh}\left(\frac{f r_{k}}{\tau_{m}}\left(\theta-\theta a 1_{j}\right)\right) \\
\sin 3_{j, k} \frac{f\left(\frac{f r_{k}}{\tau_{m}} a\right)}{\operatorname{sh}\left(\frac{f r_{k}}{\tau_{m}}\left(\theta-\theta a 2_{j}\right)\right)} \\
\left.\mathrm{L}+B 4_{j, k} \frac{f r_{k}}{\tau_{m}} a\right)
\end{array}\right) \sin \left(f r_{k} \ln \left(\frac{r}{R_{r}}\right)\right)
\end{aligned}
$$

where $f r_{k}=\frac{k \pi}{g g}, g g=\ln \left(\frac{R_{m}}{R_{r}}\right), f r a_{m}=\frac{m \pi}{a}, \theta a 1_{j}=g_{j}-\frac{a}{2}$, $\theta a 2_{j}=g_{j}+\frac{a}{2}$ and $\tau_{m}=\sqrt{\lambda_{m \theta} / \lambda_{m r}}$.
The stator yoke represented by Region III has homogenous BCs and the solution of (30) is given by

$\operatorname{TIII}(r, \theta)=A 6_{0}+A 5_{0} \ln (r)-p_{s} r^{2} /\left(4 \lambda_{s r}\right)$

$\mathrm{L}+\sum_{n=1}^{n n}\left(A 5_{n}\left(\frac{r}{R_{e x t}}\right)^{n . \tau_{s}}+A 6_{n}\left(\frac{r}{r_{4}}\right)^{-n \cdot \tau_{s}}\right) \cos (n \theta)$

$\mathrm{L}+\sum_{n=1}^{n n}\left(A 7_{n}\left(\frac{r}{R_{e x t}}\right)^{n \cdot \tau_{s}}+A 8_{n}\left(\frac{r}{r_{4}}\right)^{-n \cdot \tau_{s}}\right) \sin (n \theta)$

where $\tau_{s}=\sqrt{\lambda_{s \theta} / \lambda_{s r}}$.

The $Q_{s}$ stator slots represented by Region IVi has nonhomogenous BCs, the solution of (31) is given by

$T I V_{i}(r, \theta)=C 1_{i, 0}+C 2_{i, 0} \ln (r)-P s l_{i} r^{2} /\left(4 \lambda_{s l r}\right)$

$+\sum_{m 1=1}^{m m 1}\left(C 1_{i, m 1}\left(\frac{r}{r_{4}}\right)^{f s c_{m 1} \cdot \tau_{s l}}+C 2_{i, m 1}\left(\frac{r}{R_{s}}\right)^{-f s c_{m 1} \cdot \tau_{s l}}\right) \cos \left(f s c_{m 1}\left(\theta-\theta c 1_{i}\right)\right)$

$+\sum_{k 1=1}^{k k 1}\left(\begin{array}{c}C 3_{i, k 1} \frac{\operatorname{sh}\left(\frac{f s_{k 1}}{\tau_{s l}}\left(\theta-\theta c 1_{i}\right)\right.}{s h\left(\frac{f s_{k 1}}{\tau_{s l}} c\right)} \\ \operatorname{L~}+C 4_{i, k 1} \frac{\operatorname{sh}\left(\frac{f s_{k 1}}{\tau_{s l}}\left(\theta-\theta c 2_{i}\right)\right)}{s h\left(\frac{f s_{k 1}}{\tau_{s l}} c\right)}\end{array}\right) \sin \left(f s_{k 1} \ln \left(\frac{r}{R_{s}}\right)\right)$

where $f s_{k 1}=\frac{k 1 \pi}{f f}, f f=\ln \left(\frac{r_{4}}{R_{s}}\right), f s c_{m 1}=\frac{m 1 \pi}{c}, \theta c 1_{i}=\alpha_{i}-\frac{c}{2}$, $\theta c 2_{i}=\alpha_{i}+\frac{c}{2}$, and $\tau_{s l}=\sqrt{\lambda_{s l \theta} / \lambda_{s l r}}$.

The rotor yoke represented by Region V has homogenous BCs, the solution of (32) is given by

$$
\begin{aligned}
& T V(r, \theta)=A 10_{0}+A 9_{0} \ln (r)-p_{r} \cdot r^{2} /\left(4 \lambda_{r r}\right) \\
& \mathrm{L}+\sum_{n=1}^{n n}\left(A 9_{n}\left(\frac{r}{R_{r}}\right)^{n \cdot \tau_{r}}+A 10_{n}\left(\frac{r}{R_{i}}\right)^{-n \cdot \tau_{r}}\right) \cos (n \theta) \\
& \mathrm{L}+\sum_{n=1}^{n n}\left(A 11_{n}\left(\frac{r}{R_{r}}\right)^{n \cdot \tau_{r}}+A 12_{n}\left(\frac{r}{R_{i}}\right)^{-n \cdot \tau_{r}}\right) \sin (n \theta)
\end{aligned}
$$

where $\tau_{r}=\sqrt{\lambda_{r \theta} / \lambda_{r r}}$.

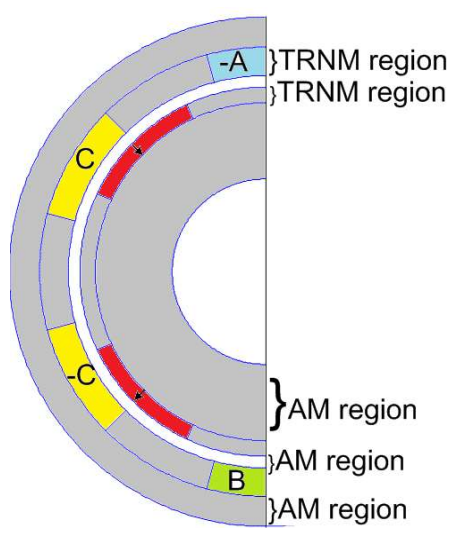

Fig. 6: Distribution of AM and TRNM regions in the HM (air-gap zoomed). 
In Region VIj with the non-homogenous BCs, the solution of (33) is given by

$$
\begin{aligned}
& \text { TVI I }_{j}(r, \theta)=B 6_{j, 0}+B 5_{j, 0} \ln (r)-P d r_{j} \cdot r^{2} /\left(4 \lambda_{a r}\right) \\
& +\sum_{m=1}^{m m}\left(B 5_{j, m}\left(\frac{r}{R_{m}}\right)^{f r b_{m} \cdot \tau_{d r}}+B 6_{j, m}\left(\frac{r}{R_{r}}\right)^{-f r b_{m} \cdot \tau_{d r}}\right) \cos \left(f r b_{m}\left(\theta-\theta b 1_{j}\right)\right) \\
& +\sum_{k=1}^{k k}\left(\begin{array}{l}
B 7_{j, k} \operatorname{sh}\left(\frac{f r_{k}}{\tau_{d r}}\left(\theta-\theta b 1_{j}\right)\right) \\
\operatorname{sh}\left(\frac{f r_{k}}{\tau_{d r}} b\right) \\
\left.\mathrm{L}+\frac{B 8_{j, k} \operatorname{sh}\left(\frac{f r_{k}}{\tau_{d r}}\left(\theta-\theta b 2_{j}\right)\right.}{\operatorname{sh}\left(\frac{f r_{k}}{\tau_{d r}} b\right)}\right)
\end{array}\right) \sin \left(f r_{k} \ln \left(\frac{r}{R_{r}}\right)\right)
\end{aligned}
$$

where $\quad f r b_{m}=\frac{m \pi}{b}, \quad \theta b 1_{j}=\beta_{j}-\frac{b}{2}, \quad \theta b 2_{j}=\beta_{j}+\frac{b}{2} \quad$ and $\tau_{d r}=\sqrt{\lambda_{d r \theta} / \lambda_{d r r}}$.

In Region VIIi representing the stator teeth with $P d s_{i}$ power losses, we have

$$
\begin{aligned}
& \operatorname{TVII}_{i}(r, \theta)=C 5_{i, 0}+C 6_{i, 0} \ln (r)-P d s_{i} \cdot r^{2} /\left(4 \lambda_{d s r}\right) \\
& +\sum_{m 1=1}^{m m 1}\left(C 5_{i, m 1}\left(\frac{r}{r_{4}}\right)^{f s d_{m 1} \cdot \tau_{d s}}+C 6_{i, m 1}\left(\frac{r}{R_{s}}\right)^{-f s d_{m 1} \cdot \tau_{d s}}\right) \cos \left(f s d_{m 1}\left(\theta-\theta d 1_{i}\right)\right) \\
& +\sum_{k 1=1}^{k k 1}\left(\begin{array}{c}
\operatorname{sh}\left(\frac{f s_{k 1}}{\tau_{d s}}\left(\theta-\theta d 1_{i}\right)\right) \\
\operatorname{sh}\left(\frac{f s_{k 1}}{\tau_{d s}} d\right) \\
\left.\mathrm{L}+C 8_{i, k 1} \frac{\operatorname{sh}\left(\frac{f s_{k 1}}{\tau_{d s}}\left(\theta-\theta d 2_{i}\right)\right.}{\operatorname{sh}\left(\frac{f s_{k 1}}{\tau_{d s}} d\right)}\right)
\end{array}\right) \sin \left(f s_{k 1} \ln \left(\frac{r}{R_{s}}\right)\right)
\end{aligned}
$$

where $f_{s_{k 1}}=\frac{k 1 \pi}{f f}, f f=\ln \left(\frac{r_{4}}{R_{s}}\right), f_{s} d_{m 1}=\frac{m 1 \pi}{d}, \theta d 1_{i}=\gamma_{i}-\frac{d}{2}$, $\theta d 2_{i}=\gamma_{i}+\frac{d}{2}$ and $\tau_{d s}=\sqrt{\lambda_{d s \theta} / \lambda_{d s r}}$.

The anisotropy coefficient can be defined as

$$
\psi=\lambda_{\theta} / \lambda_{r}
$$

\section{3-ICs and their Development}

To determine the unknown coefficients of temperature in each $\mathrm{SD}$, there are $18 \mathrm{ICs}$, viz., $14 \mathrm{ICs}$ are in the $\theta$-direction and 4 ICs in the $r$-direction [7]. The development of ICs permits to obtain an equations system whose unknowns are the coefficients of Fourier's series solution in each SD. The solving of this system gives the temperature and heat flux distribution in the whole machine.

For the studied inset-PM machine with the harmonics number in each SD: $m m l=40, k k l=40, m m=50, k k=50$ and $n n=200$, the dimensions of global matrix and vector are

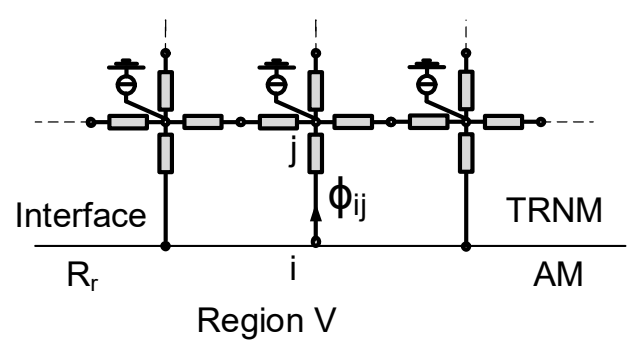

Fig. 7: Heat flux density IC between TRNM and AM at $R_{r}$.

\section{Hybrid Method (HM)}

It is well known that AM is more accurate with low computational time than TRNM. However, when the number of SDs is high (i.e., the ICs number in both directions is also high) and their dimensions are small, the harmonics number can be very low and then the accuracy of AM can be very low. This situation can be found in the case of inset-PM machine with high number of stator and rotor slots. For this, we propose in this paper to model the stator and rotor slots regions (also the stator and rotor teeth) with TRNM and the other regions with AM [see Fig. 6].

To release the coupling between AM and TRNM, nodes of coupling are added to TRNM presented in Section A at the radii separating the two methods (ICs between AM and TRNM at $R_{r}, R_{m}, R_{s}$ and $r_{4}$ ).

For example, at the radius $R_{r}$, the continuity conditions of AM are given by

$$
\begin{aligned}
& T V\left(R_{r}, \theta\right)=T I I_{j}\left(R_{r}, \theta\right) \\
& T V\left(R_{r}, \theta\right)=T V I_{j}\left(R_{r}, \theta\right) \\
& q V_{r}\left(R_{r}, \theta\right)= \begin{cases}q I I_{r, j}\left(R_{r}, \theta\right) & \text { for } \theta \in\left[g_{j}+\frac{a}{2} ; g_{j}-\frac{a}{2}\right] \\
q V I_{r, j}\left(R_{r}, \theta\right) & \text { for } \theta \in\left[\beta_{j}+\frac{b}{2} ; \beta_{j}-\frac{b}{2}\right]\end{cases}
\end{aligned}
$$

In the HM, the Region IIj and VIj are modeled by TRNM and Region V by AM. To satisfy the ICs (57) and (58), the nodes temperatures of TRNM at $R_{r}$ are written as a $2 \pi$ periodic function using discrete Fourier series [12] as

$$
\begin{aligned}
& T_{\text {TRNM_}_{-} R}(\theta)=\frac{a_{0}}{2}+\sum_{n=1}^{n n} a_{n} \cos (n \theta)+b_{n} \sin (n \theta) \\
& a_{0}=\frac{2}{2 \cdot n n} \cdot \sum_{k=n o d b(1,1,1)}^{\operatorname{nodb}(1,1, n s)} T_{k} \\
& a_{n}=\frac{2}{2 \cdot n n} \cdot \sum_{k=n o d b(1,1,1)}^{n o d b(1,1, n s)} T_{k} \cos \left(n \theta_{k}\right) \quad \forall n \\
& b_{n}=\frac{2}{2 \cdot n n} \cdot \sum_{k=n o d b(1,1,1)}^{n o d b(1,1, n s)} T_{k} \sin \left(n \theta_{k}\right) \quad \forall n
\end{aligned}
$$

where $n n=n s / 2, T_{k}$ are the temperatures at the boundary nodes $\operatorname{nodb}(1, i, 1)$ to $\operatorname{nodb}(1, i, n s)$ situated at $R_{r}(i=1)$ and 
$\theta_{k}$ the angular position of boundary nodes at the radius $R_{r}$. It is important to note that $n n$ is also the total harmonics number of the AM solution in the Region V.

This Fourier series function permits to replace the ICs (57) and (58) by

$$
T V\left(R_{r}, \theta\right)=T_{\text {TRNM_Rr }_{-}}(\theta)
$$

This above equation permits to get 3 equations as

$$
\begin{aligned}
& -\frac{1}{4} \frac{q_{r} R_{r}^{2}}{\lambda_{r r}}+A 9_{0} \ln \left(R_{r}\right)+A 10_{0}=\frac{1}{2 \cdot n n} \cdot \sum_{k=n o d b(1,1,1)}^{n o d b(1,1, n s)} T_{k} \\
& A 9_{n}+A 10_{n}\left(\frac{R_{r}}{R_{i}}\right)^{-n \cdot \tau_{r}}=\frac{1}{n n} \sum_{k=n o d b(1,1,1)}^{\operatorname{nodb}(1,1, n s)} T_{k} \cos \left(n \theta_{k}\right) \\
& A 11_{n}+A 12_{n}\left(\frac{R_{r}}{R_{i}}\right)^{-n \cdot \tau_{r}}=\frac{1}{n n} \sum_{k=n o d b(1,1,1)}^{\operatorname{nodb}(1,1, n s)} T_{k} \sin \left(n \theta_{k}\right)
\end{aligned}
$$

To satisfy the heat flux density IC at $R_{r}$, i.e., (59), each boundary node is considered receiving a radial heat flux $\phi_{i j}$ from the AM region [see Fig. 7] as [20]-[21]

$$
\phi_{i j}=\frac{T_{i}-T_{j}}{R_{i j}}=-\lambda_{r r} R_{r} L_{u} \int_{\theta_{k}-d \theta}^{\theta_{k}+d \theta} \frac{\partial T V(r, \theta)}{\partial r} d \theta
$$

where $\theta_{k}$ is the angular position of interface nodes and $d \theta$ is half opening angle of an element equal to $0.5^{\circ}$ (each element of TRNM has an angular opening equal $1^{\circ}$ in TRNM).

The development of (65) gives

$$
\begin{aligned}
& \phi_{i j}=\left(-L_{u} \lambda_{r r} A 9_{0}+\frac{R_{r}^{2}}{2} L_{u} q_{r} \int_{\theta_{k}-d \theta}^{\theta_{k}+d \theta} d \theta\right. \\
& \cdots+L_{u} \lambda_{r r} n \tau_{r}\left(-A 9_{n}+A 10_{n}\left(\frac{R_{r}}{R_{i}}\right)^{-n \tau_{r}}\right) \int_{\theta_{k}-d \theta}^{\theta_{k}+d \theta} \cos (n \theta) d \theta \\
& \cdots+L_{u} \lambda_{r r} n \tau_{r}\left(-A 11_{n}+A 12_{n}\left(\frac{R_{r}}{R_{i}}\right)^{-n \tau_{r}}\right) \int_{\theta_{k}-d \theta}^{\theta_{k}+d \theta} \sin (n \theta) d \theta
\end{aligned}
$$

In the HM, $m s=21$ and $n s=360$ with 11 radii for TRNM rotor region and 11 radii for TRNM stator region. The elements and nodes number of TRNM stator region is $n s g s=3,600$ and TRNM rotor region is $n s g r=3,600$. In TRNM rotor region, we have added 2 boundaries additional nodes at $R_{r}(i=1)$ and $R_{m}(i=11) \quad$ represented by vectors $\operatorname{nodb}(1,1, j)$ and $\operatorname{nodb}(2,11, j)$ where $j$ varies from 1 to $n s$. For TRNM stator region, we have added 2 boundaries nodes at $R_{s}(i=12)$ and $r_{4}(i=22)$ represented by vectors $\operatorname{nodb}(3,12, j)$ and $\operatorname{nod} b(4,22, j)$. The nodes of TRNM connected to the 4 boundary nodes $\operatorname{nodb}(1,1, j), \operatorname{nodb}(2,11, j), \operatorname{nodb}(3,12, j)$,

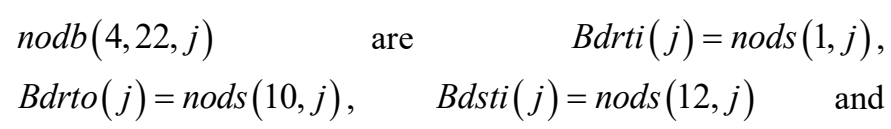

$\operatorname{Bdsto}(j)=\operatorname{nods}(m s, j)$ respectively. The total elements and nodes number of TRNM mesh considering the nodes number at the $4 \mathrm{BCs}$ is equal to $n s g b=8,640$. There are $3 \mathrm{AM}$ regions in the $\mathrm{HM}$ where each $\mathrm{AM}$ region necessitates $2+4 \cdot n n$ unknowns. The total nodes number of TRNM regions and AM regions is $n s g b 1=10,806$.

After defining the nodes connectivity as described in Section A, the assembly of the global matrix from TRNM and AM is performed. We start with the TRNM nodes representing the rotor slots and teeth. The introduction of equations (62) to (64) in the global matrix $\mathrm{gm}$ is performed as given in Appendix B.

There are 360 equations (66) to be introduced in the global matrix depending on the number of boundary nodes at $R_{r}$. The equations (62) to (64) and (66) related to the ICs (57) to (59) at $R_{r}$ have been introduced in the global matrix $g m$ and the global vector $f$ of the HM. The coupling is performed between the AM temperature of rotor $T V(r, \theta)(53)$ and TRNM at $R_{r}$. In the same way, the coupling between $T I(r, \theta)(48)$ and rotor TRNM at $R_{m}$ and $R_{s}$, and the coupling between $\operatorname{TIII}(r, \theta)$ (50) and stator TRNM at $r_{4}$ can be established and assembled to the global system. The introduction of convection heat transfer at the external radius and shaft is done using AM.

TABLE. I

PARAMETERS OF STUDIED INSET-PM MACHINE.

\begin{tabular}{ccc}
\hline \hline Symbol & Parameters & Value \\
\hline$B_{r m}$ & Remanence flux density of PMs & $1.3 \mathrm{~T}$ \\
$\mu_{r m}$ & Relative permeability of PMs & 1.0277 \\
$N_{c}$ & Number of conductors per stator slot & 23 \\
$I_{m}$ & Peak phase current & $7 \mathrm{~A}$ \\
$Q_{s}$ & Number of stator slots & 6 \\
$c$ & Stator slot-opening & $30 \mathrm{deg}$. \\
$a$ & PM-opening & $40 \mathrm{deg}$. \\
$p$ & Number of pole pairs & 2 \\
$R_{e x t}$ & Radius of the external stator surface & $110 \mathrm{~mm}$ \\
$r_{4}$ & Outer radius of stator slot & $97 \mathrm{~mm}$ \\
$R_{s}$ & Radius of the stator inner surface & $80.5 \mathrm{~mm}$ \\
$R_{m}$ & Radius of the rotor outer surface at the PM & $79.7 \mathrm{~mm}$ \\
$R_{r}$ & Radius of the rotor inner surface at the PM & $73 \mathrm{~mm}$ \\
$g$ & Air-gap length & $0.8 \mathrm{~mm}$ \\
$L_{u}$ & Axial length & $40 \mathrm{~mm}$ \\
$\Omega$ & Mechanical speed & $500 \mathrm{rmm}$ \\
\hline \hline
\end{tabular}

TABLE. II

PARAMETERS OF THE THERMAL MODEL.

\begin{tabular}{ccc}
\hline \hline Symbol & Parameters & Value \\
\hline$\lambda_{e}$ & Thermal conductivity of air-gap & $0.03 \mathrm{~W} /(\mathrm{m} \mathrm{K})$ \\
$\lambda_{a}$ & Thermal conductivity of air & $0.03 \mathrm{~W} /(\mathrm{m} \mathrm{K})$ \\
$\lambda_{m}$ & Thermal conductivity of PMs & $9 \mathrm{~W} /(\mathrm{m} \mathrm{K})$ \\
$\lambda_{s}$ & Thermal conductivity of stator iron & $55 \mathrm{~W} /(\mathrm{m} \mathrm{K})$ \\
$\lambda_{r}$ & Thermal conductivity of rotor iron & $55 \mathrm{~W} /(\mathrm{m} \mathrm{K})$ \\
$\lambda_{s l}$ & Thermal conductivity of stator slot coil & $1.73 \mathrm{~W} /(\mathrm{m} \mathrm{K})$ \\
$p_{s}$ & Stator core losses & $4.07 \mathrm{~W}$ \\
$p_{r}$ & Rotor core losses & $0.31 \mathrm{~W}$ \\
$p_{m}$ & PM losses & $7.94 \mathrm{~W}$ \\
$p_{s l}$ & Stator slot losses & $18.12 \mathrm{~W}$ \\
$P_{e}$ & Windage losses in the air-gap & $5 \mathrm{~W}$ \\
$h_{r}$ & Convection coefficient inside the rotor & $100 \mathrm{~W} /\left(\mathrm{m}^{2} \mathrm{~K}\right)$ \\
$T_{i n t}$ & Temperature inside the rotor & $70{ }^{\circ} \mathrm{C}$ \\
$h_{s}$ & Convection coefficient outside the stator & $100 \mathrm{~W} /\left(\mathrm{m}^{2} \mathrm{~K}\right)$ \\
$T_{\text {ext }}$ & Temperature outside the stator & $70{ }^{\circ} \mathrm{C}$ \\
\hline
\end{tabular}




\begin{tabular}{ccc}
\hline$\psi$ & Anisotropy coefficients & $0.5 / 1 / 1.5$ \\
\hline
\end{tabular}

\section{TEMPERATURE AND HEAT FLUX RESULTS}

The parameters and dimensions of the studied inset-PM machine are given in Table I. The machine has a simple distributed 4 poles winding. The power losses of the inset-PM machine at $500 \mathrm{rpm}$ as well as the thermal conductivities, convection coefficients and ambient temperatures used in the thermal model are listed in Table II. The harmonics number of $\mathrm{AM}$ is $n n=200, m m=50, k k=50, m m 1=40$ and $k k 1=40$ . These harmonics numbers provide very good accuracy compared to Fem with a reasonable computation time. The average elements and nodes number of the Fem calculation [15] are respectively 109,168 and 55,484.

\section{A. TRNM Results Without Materials Anisotropy and Validation with Fem}

For the 6-slots/4-poles inset-PM machine, the temperature distribution at speed of $500 \mathrm{rpm}$ in the whole machine without taking into account the materials anisotropy is shown in Fig. 8. We can observe that the temperature is higher inside the stator slots where power loss is higher [see Table II]. The directions of heat flux are represented with vectors oriented to inside and outside the machine. This is due to convections coefficients imposed outside and inside the machine. In the middle of the airgap, the distribution of temperature and heat flux components calculated by the developed TRNM and Fem using the parameters and power losses in Table II are given in Fig. 9.

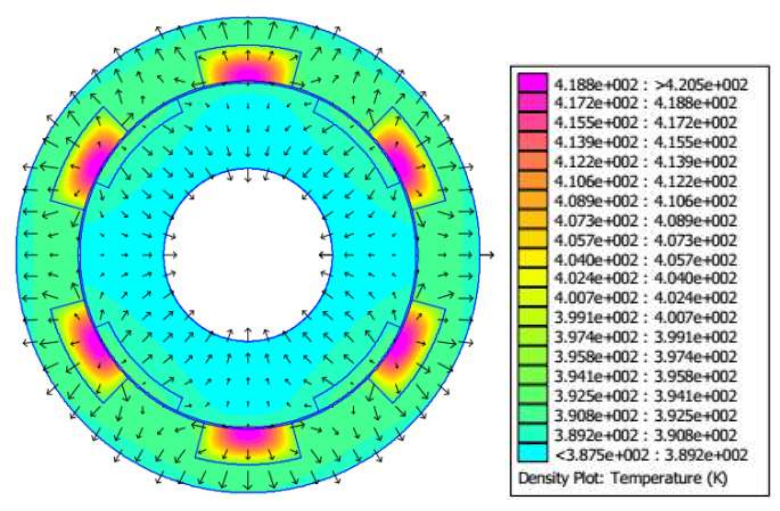

Fig. 8: Temperature and flux distribution obtained using Fem.

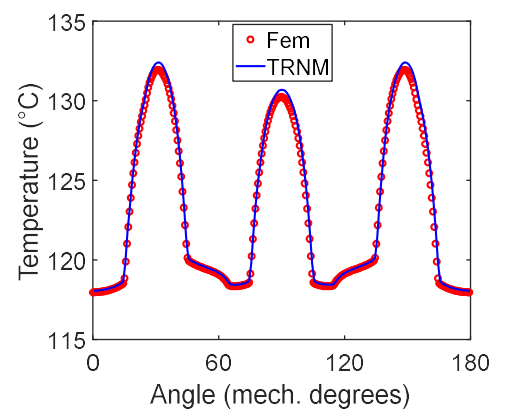

(a)

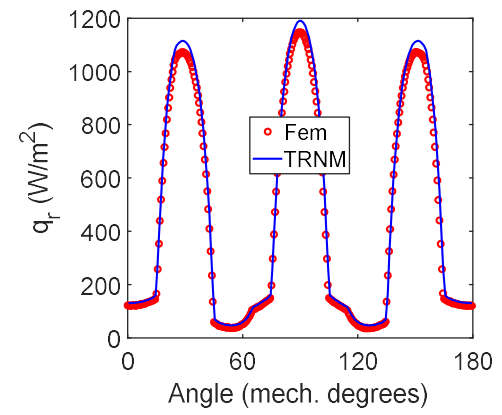

(b)

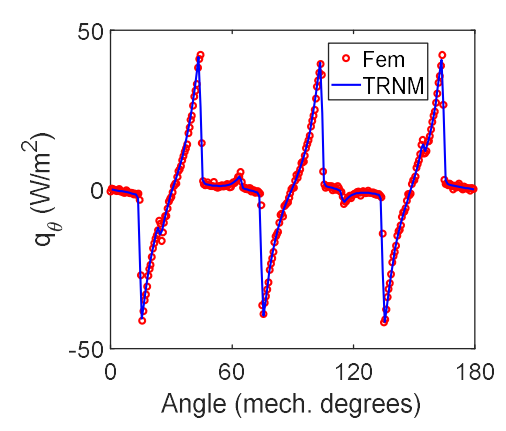

(c)

Fig. 9: Temperature and heat flux components distribution in the air-gap at the radius $80.05 \mathrm{~mm}$.

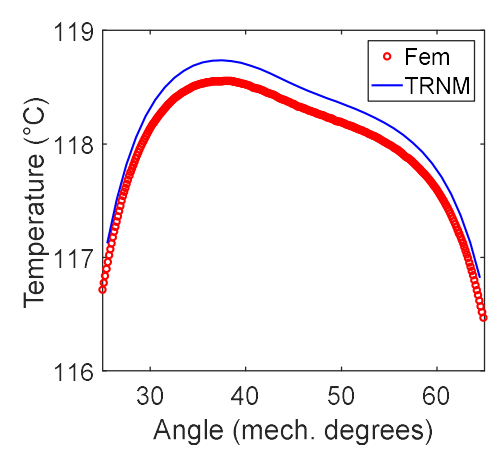

(a) In the $\theta$-direction at $76.77 \mathrm{~mm}$.

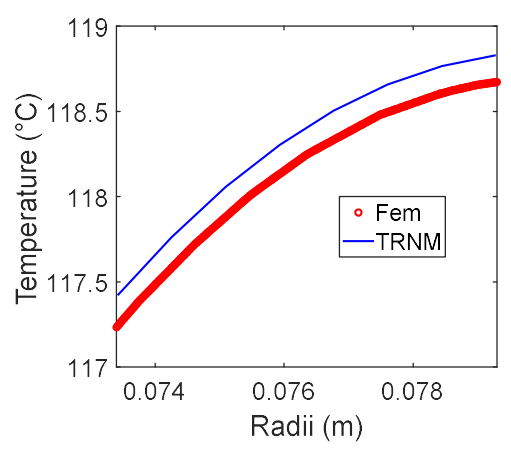

(b) In the $r$-direction at 45.5 degrees.

Fig. 10: Temperature in the middle of the first PM.

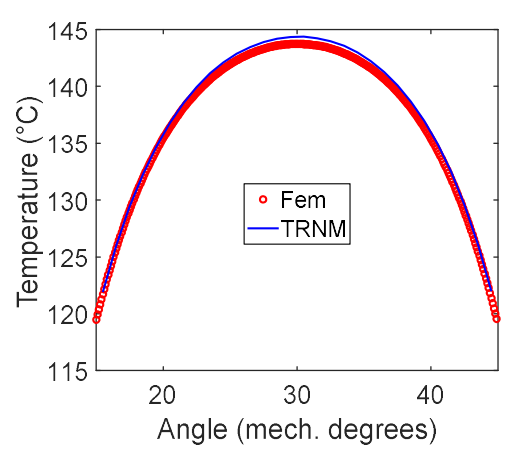

(a) In the $\theta$-direction at $87.72 \mathrm{~mm}$. 


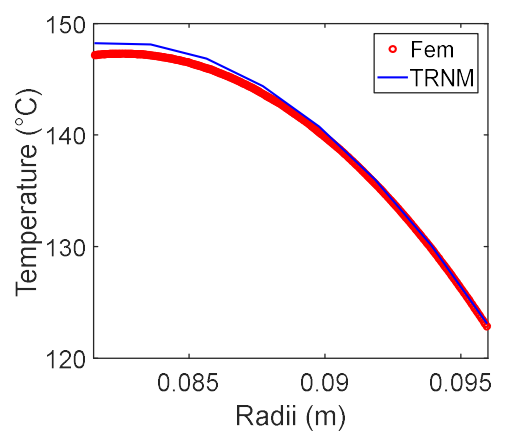

(b) In the $r$-direction at 30.5 degrees.

Fig. 11: Temperature in the middle of the first stator slot.

To show the ability of the TRNM to predict the temperature distribution in the PMs and the stator slots, the temperature curves in the $\theta$ - and $r$-direction obtained using TRNM are shown in Figs. $10 \sim 11$ and compared with Fem. The TRNM results are in good agreement with the Fem results. A small difference exist between TRNM and Fem results in the PM region. This difference is due to the meshing of machine which can be improved as done in TRNM [22]-[23]. A parametric analysis with variation of the convective coefficients $h_{s}$ and $h_{r}$ is also performed. Fig. 12 shows the temperature and heat flux distribution in the inset-PM machine when the convective coefficients $h_{s}=20 \mathrm{~W} /\left(\mathrm{m}^{2} \cdot K\right)$ and $h_{r}=100 \mathrm{~W} /\left(\mathrm{m}^{2} \cdot \mathrm{K}\right)$. The vecors of heat flux are oriented to inside and outside the machine. It can be seen that the heat flux oriented to inside the rotor is higher than the heat flux oriented to outside the stator. The corresponding air-gap temperature distribtion is shown in Fig. 13. It can be observed that the temperature is higher than in the case with $h_{s}=100 \mathrm{~W} /\left(\mathrm{m}^{2} \cdot \mathrm{K}\right)$. For the case with $h_{r}=20 \mathrm{~W} /\left(\mathrm{m}^{2} \cdot \mathrm{K}\right)$ and $h_{s}=100 \mathrm{~W} /\left(\mathrm{m}^{2} \cdot \mathrm{K}\right)$ , Figs. $14 \sim 15$ show the temperature distribution in the machine obtained using Fem and temperature distribution in the middle of the air-gap obtained using the developed TRNM and Fem. It can be seen from Fig. 14 that the heat flux oriented to outside the stator is higher than the heat flux oriented to inside the rotor. Also, in this case, the TRNM results are very close to those of Fem. The variation of the temperature in the middle of the PM and stator slot when the convective coefficients $h_{s}$ and $h_{r}$ varies is shown in Figs. $16 \sim 17$. The comparison of the TRNM results with those obtained by Fem confirms the validity of the proposed TRNM to predict the temperature and heat flux distribution in the inset-PM machine with a very good accuracy.

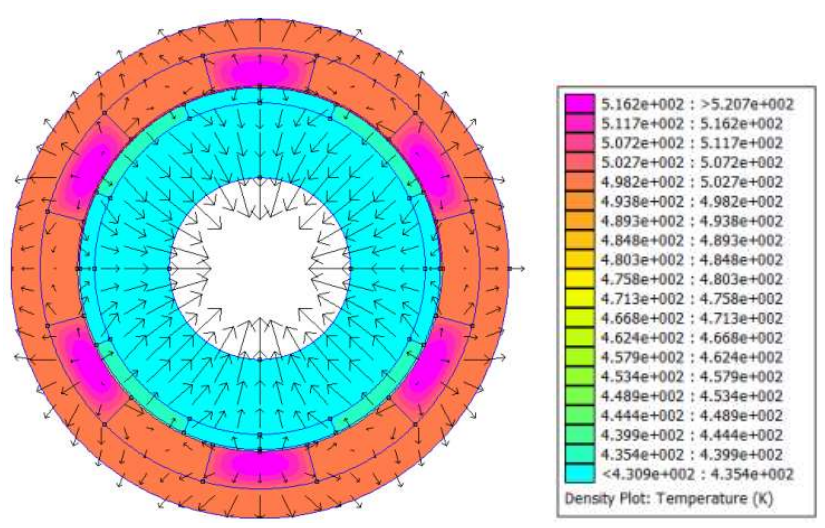

Fig. 12: Temperature distribution and heat flux in the inset-PM machine for $h_{s}=20 \mathrm{~W} /\left(\mathrm{m}^{2} \cdot \mathrm{K}\right)$ and $h_{r}=100 \mathrm{~W} /\left(\mathrm{m}^{2} \cdot K\right)$.

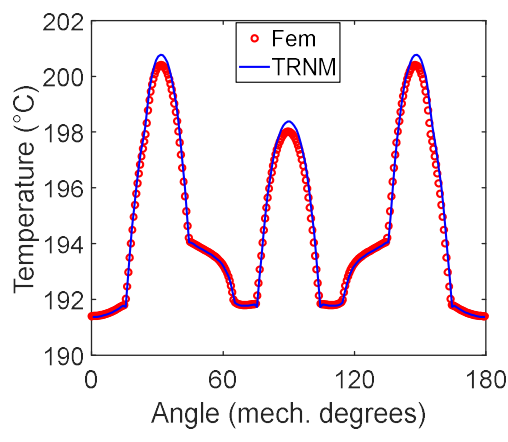

Fig. 13: Temperature distribution in the middle of the air-gap for $h_{s}=20 \mathrm{~W} /\left(\mathrm{m}^{2} \cdot K\right)$ and $h_{r}=100 \mathrm{~W} /\left(\mathrm{m}^{2} \cdot K\right)$.

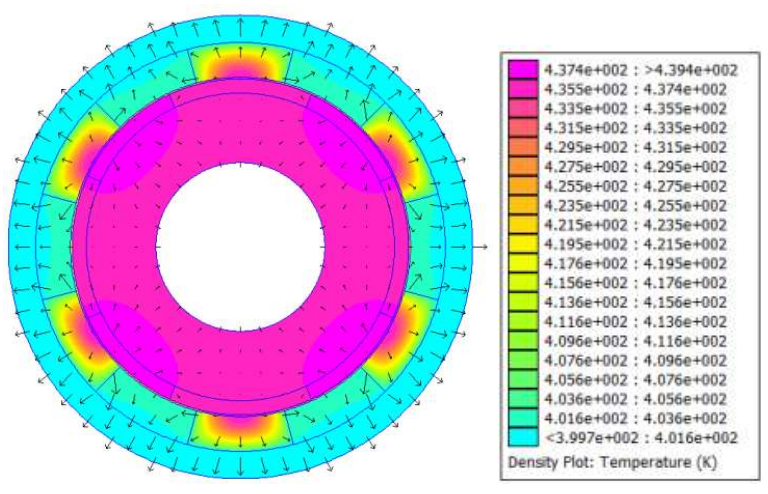

Fig. 14: Temperature and heat flux distribution in the inset-PM machine for $h_{r}=20 \mathrm{~W} /\left(\mathrm{m}^{2} \cdot K\right)$ and $h_{s}=100 \mathrm{~W} /\left(\mathrm{m}^{2} \cdot K\right)$.

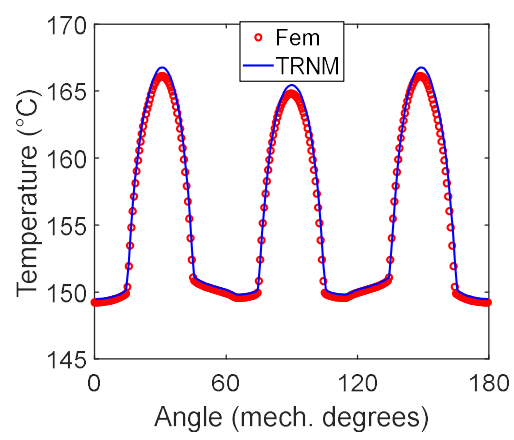

Fig. 15: Temperature distribution in the middle of air-gap for $h_{r}=20 \mathrm{~W} /\left(\mathrm{m}^{2} \cdot K\right)$ and $h_{s}=100 \mathrm{~W} /\left(\mathrm{m}^{2} \cdot K\right)$.

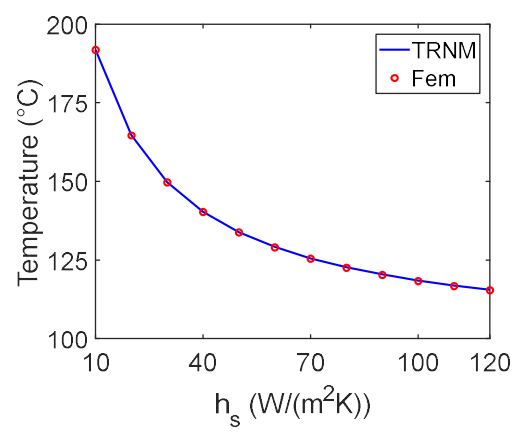

(a) Temperature at the center of the first PM.

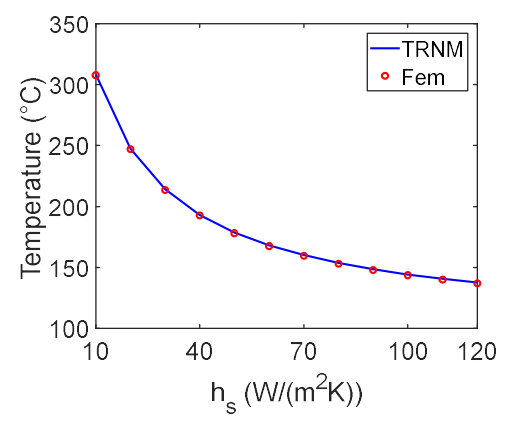


(b) Temperature at the center of the first stator slot.

Fig. 16: Temperature variation with varying $h_{s}$ and $h_{r}=100 \mathrm{~W} /\left(\mathrm{m}^{2} \cdot \mathrm{K}\right)$ in a point at the center of PM and stator slot.

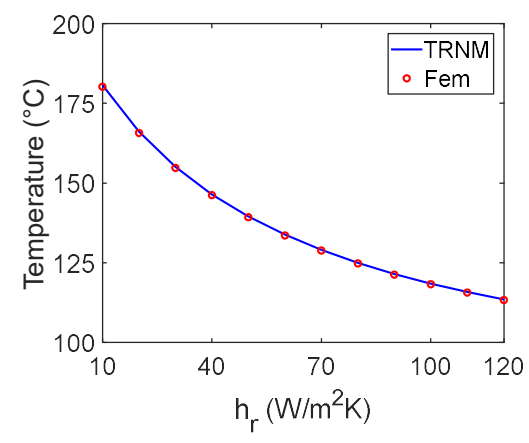

(a) Temperature at the center of the first PM.

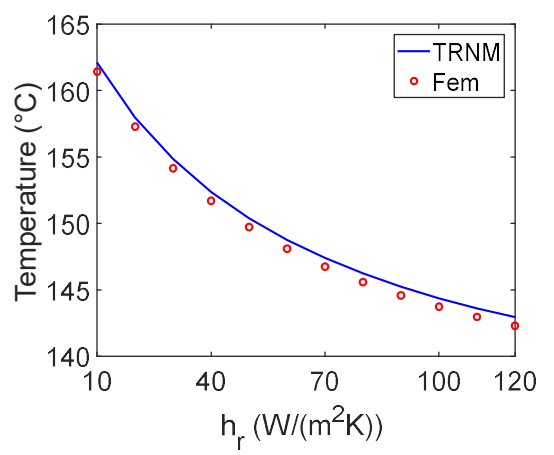

(b) Temperature at the center of the first stator slot.

Fig. 17: Temperature variation with varying $h_{r}$ and $h_{s}=100 \mathrm{~W} /\left(\mathrm{m}^{2} \cdot K\right)$ in a point at the center of PM and stator slot.

\section{B. AM Thermal Results with Materials Anisotropy and Validation with TRNM}

The distribution of temperature and heat flux components in the middle of the air-gap obtained with AM and TRNM taking into account the materials anisotropy is shown in Fig. 18. We can observe a very good agreement between the AM and TRNM results. The temperature distribution in the middle of the first PM and the stator slot in the $\theta$ - and $r$-direction [see Figs. $19 \sim 20$ ] obtained analytically and with TRNM confirm the accuracy of the proposed AM. Again, we can show a small difference due to the mesh size adopted in TRNM.

When the cooling outside the inset-PM machine is not sufficient, i.e., $h_{s}=20 \mathrm{~W} /\left(\mathrm{m}^{2} \cdot \mathrm{K}\right)$, the heat is not evacuated and the temperature is very high in the air-gap [see Fig. 21]. The same observation can be done in the case of insufficient cooling in the rotor shaft with $h_{r}=20 \mathrm{~W} /\left(\mathrm{m}^{2} \cdot K\right)$ [see Fig. 22]. In this case, the rotor temperature is high but lower than the case of low value of $h_{s}$. The variation of temperature in the middle of the first PM and the stator slot with the convection coefficient $h_{s}$ and $h_{r}$ is shown in Figs. $23 \sim 24$. Those curves are very important for the design of stator winding insulation and PMs whose characteristics depend on temperature.

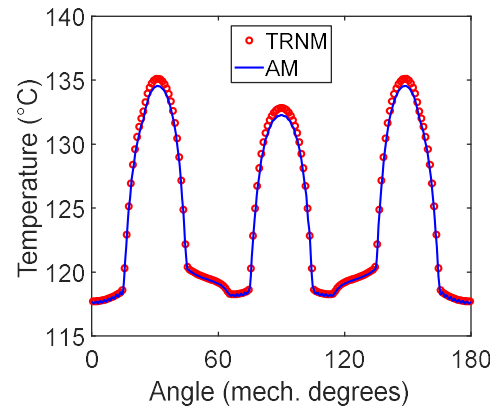

(a)

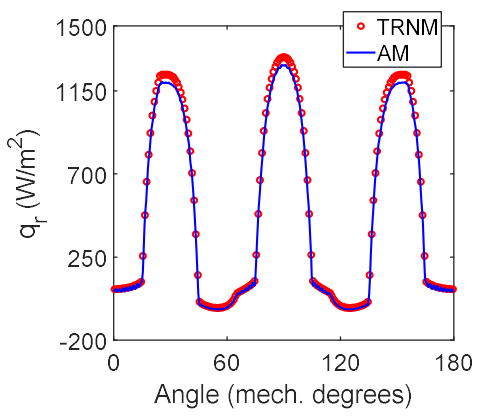

(b)

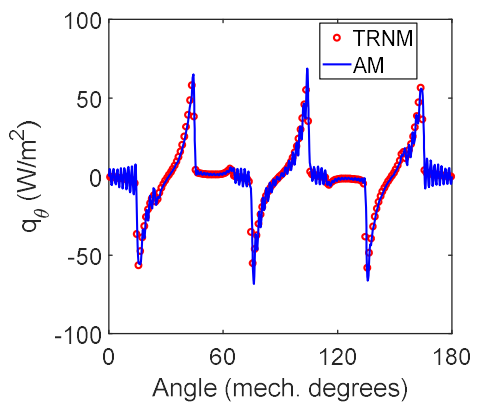

(c)

Fig. 18: Temperature and heat flux components distribution in the middle of the air-gap.

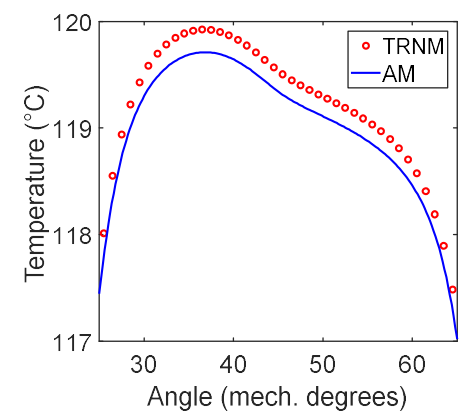

(a) In the $\theta$-direction.

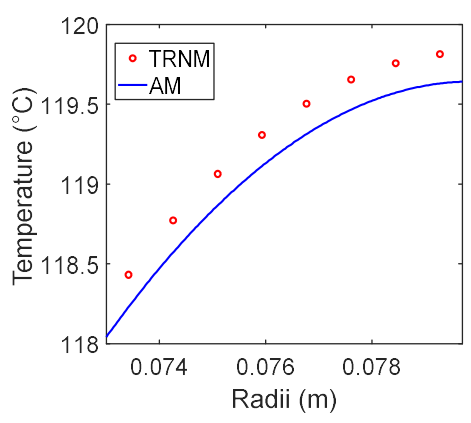

(b) In the $r$-direction 
Fig. 19: Temperature distribution in the middle of the first PM.

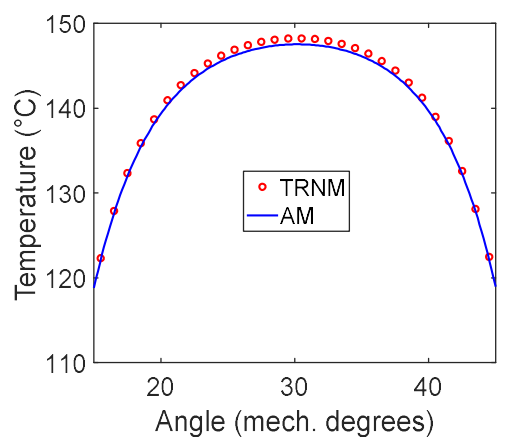

(a) In the $\theta$-direction.

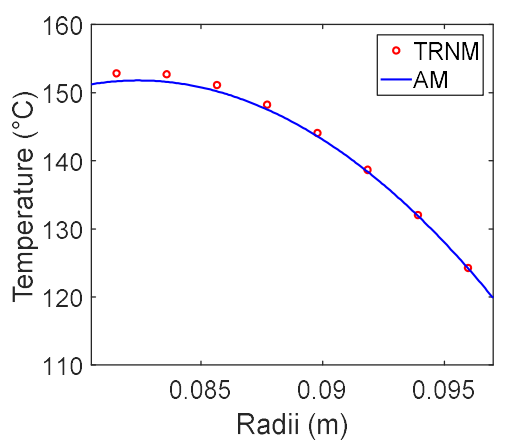

(b) In the $r$-direction.

Fig. 20: Temperature in the middle of the first stator slot.

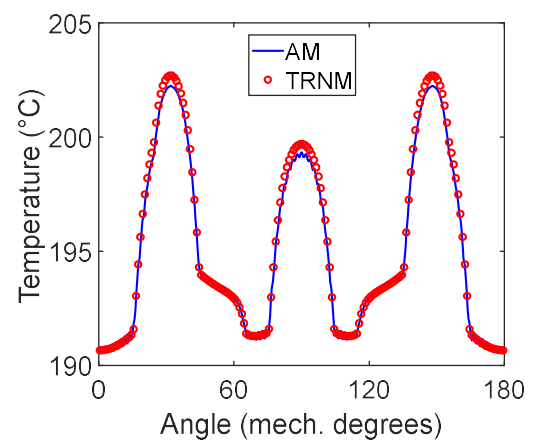

Fig. 21: Temperature distribution in the middle of the air-gap for $h_{s}=20 \mathrm{~W} /\left(\mathrm{m}^{2} \cdot K\right)$ and $h_{r}=100 \mathrm{~W} /\left(\mathrm{m}^{2} \cdot K\right)$.

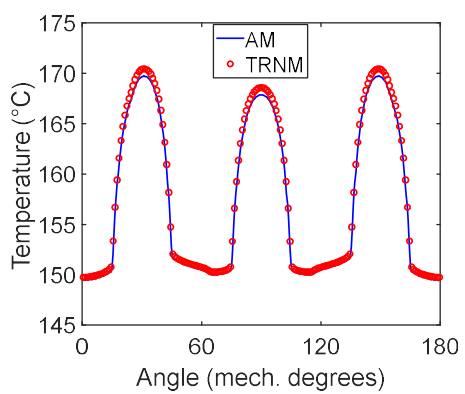

Fig. 22: Temperature distribution in the middle of the air-gap for $h_{r}=20 \mathrm{~W} /\left(\mathrm{m}^{2} \cdot K\right)$ and $h_{s}=100 \mathrm{~W} /\left(\mathrm{m}^{2} \cdot K\right)$.

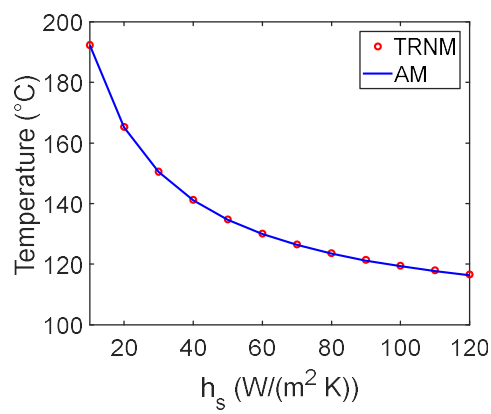

(a) Temperature at the center of the first PM.

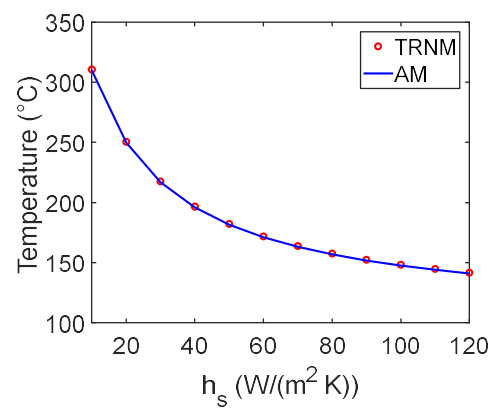

(b) Temperature at the center of the first stator slot.

Fig. 23: Temperature variation with varying $h_{s}$ and $h_{r}=100 \mathrm{~W} /\left(\mathrm{m}^{2} \cdot K\right)$ in a point at the center of PM and stator slot.

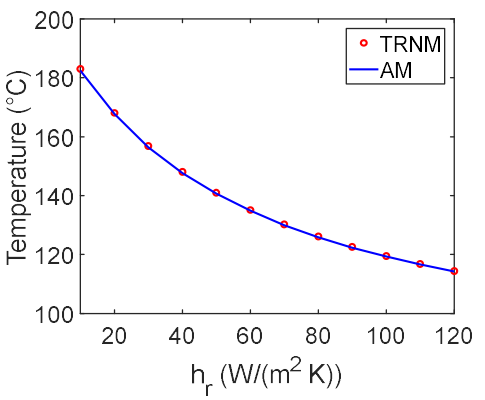

(a) Temperature at the center of the first PM.

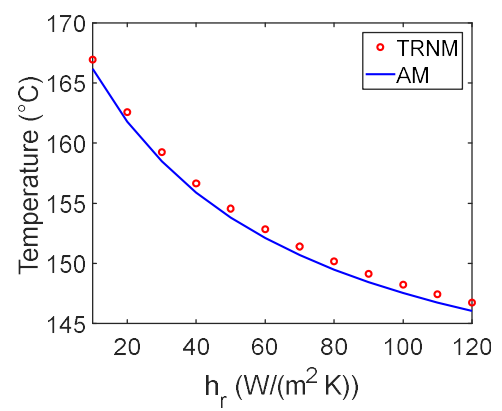

(b) Temperature at the center of the first stator slot.

Fig. 24: Temperature variation with varying $h_{r}$ and $h_{s}=100 \mathrm{~W} /\left(\mathrm{m}^{2} \cdot K\right)$ in a point at the center of PM and stator slot.

\section{HM Thermal Results and Validation with TRNM and Fem}

It is not easy to use AM in heat transfer prediction for rotating electrical machines with high number of stator slots and rotor poles. The ICs in the $r$ - and $\theta$-direction are important and the dimensions of SDs are small, requiring a small harmonics number and thus lower accuracy. For this, it is appropriate to use HM. In this section, we apply the HM in both cases, with isotropic and anisotropic materials. For isotropic materials, the validation of results can be performed with Fem.

\section{1- Isotropic Materials}

The temperature and heat flux distribution in the middle of the air-gap is shown in Fig. 25. The HM results are very close to those from Fem. The temperature distribution in the $\theta$ - and $r$ direction in the middle of the first PM and the first stator slot is shown in Figs. $26 \sim 27$. The accuracy of HM is established also in those SDs where it is important to know the heat transfer for the insulation design. A small difference can be observed in the PM region between HM and Fem. The mesh size of the TRNM rotor has affected the HM results. For this, it is necessary to optimize the TRNM parts using them in HM. 
The effect of cooling outside the inset-PM machine and inside the rotor shaft is represented with the convective coefficients $h_{s}$ and $h_{r}$ respectively. In Fig. 28, for $h_{s}=20 \mathrm{~W} /\left(\mathrm{m}^{2} \cdot K\right)$ which is small, we represent the temperature distribution in the middle of the air-gap. The temperature is higher compared to $h_{s}=100 \mathrm{~W} /\left(\mathrm{m}^{2} \cdot \mathrm{K}\right)$.

For $h_{r}=20 \mathrm{~W} /\left(\mathrm{m}^{2} \cdot K\right)$ compared to $h_{r}=100 \mathrm{~W} /\left(\mathrm{m}^{2} \cdot K\right)$, the temperature distribution in the air-gap [see Fig. 29] is higher than the case with $h_{r}=100 \mathrm{~W} /\left(\mathrm{m}^{2} \cdot \mathrm{K}\right)$. Low values of convective coefficients represent a barrier for heat transfer outside the stator and inside the rotor.

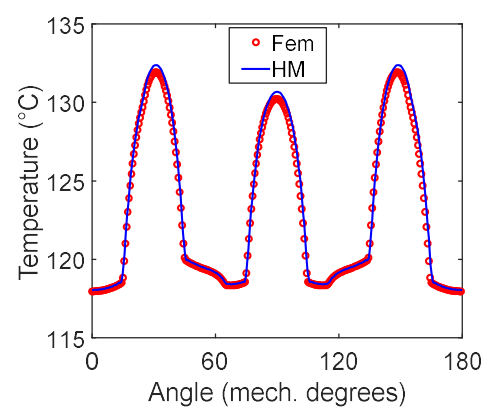

(a)

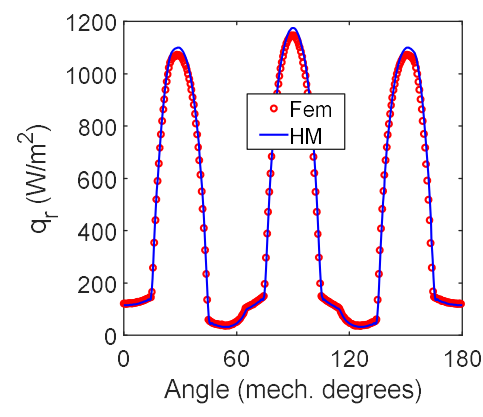

(b)

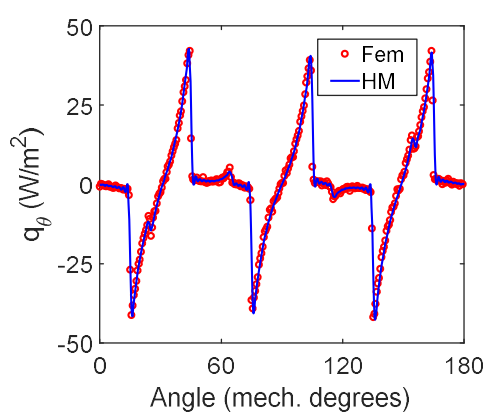

(c)

Fig. 25: Temperature and heat flux components distribution in the middle of the air-gap.

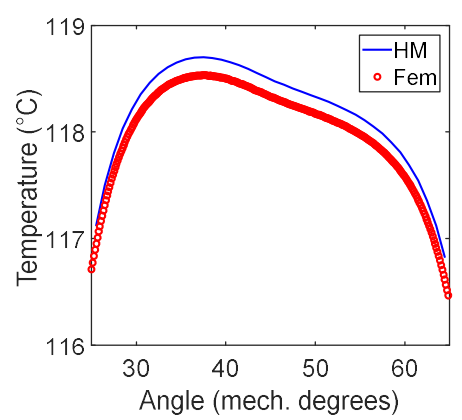

(a) In the $\theta$-direction.

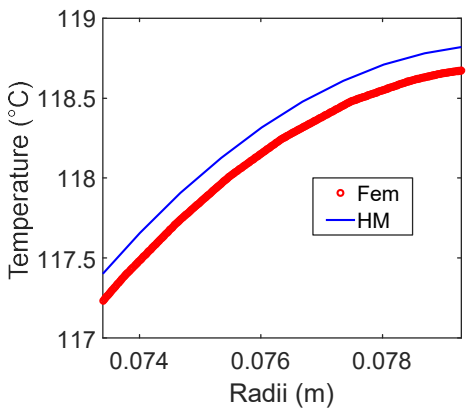

(b) In the $r$-direction

Fig. 26: Temperature in the middle of the first PM.

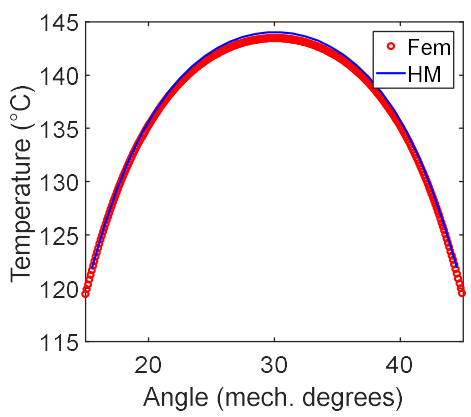

(a) In the $\theta$-direction

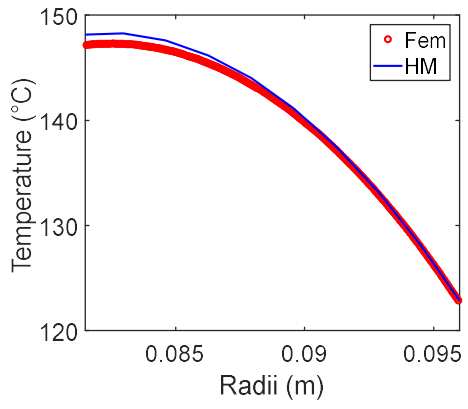

(b) In the $r$-direction.

Fig. 27: Temperature in the middle of the first stator slot.

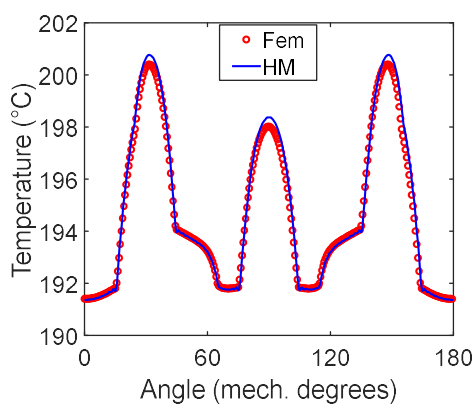

Fig. 28: Temperature distribution in the middle of the air-gap for $h_{s}=20 \mathrm{~W} /\left(\mathrm{m}^{2} \cdot K\right)$ and $h_{r}=100 \mathrm{~W} /\left(\mathrm{m}^{2} \cdot K\right)$.

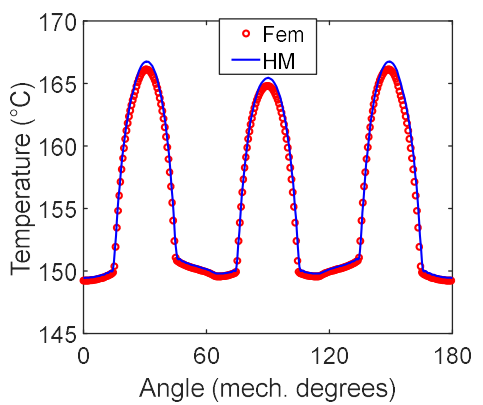

Fig. 29: Temperature distribution in the middle of the air-gap for $h_{r}=20 \mathrm{~W} /\left(\mathrm{m}^{2} \cdot \mathrm{K}\right)$ and $h_{s}=100 \mathrm{~W} /\left(\mathrm{m}^{2} \cdot \mathrm{K}\right)$. 


\section{2- Anisotropic Materials}

In the analysis of heat transfer in the inset-PM machine taking into account the materials anisotropy, TRNM is used for the validation of results. This is due to Fem which use Cartesian representation of thermal conductivities [17] and [19]. For the dimensions and parameters of the studied machine [see Tables I and II] and an anisotropy coefficient equal to 0.5 , we represent on Fig. 30 the distribution of temperature and heat flux in the middle of the air-gap. It can be seen that the temperature in the air-gap is higher than in the case of isotropic materials and the comparison between the two methods gives very good agreement. It is important to note that the anisotropy coefficient of materials is applied for rotor and stator iron, slots and PM without air-gap.

A parametric study is performed in this section as a function of the anisotropy coefficient (viz., $\psi=0.5,1,1.5$ ). When $\psi=1$, the materials are isotropic and when $\psi=0.5$ the tangential value of thermal conductivity is smaller than the radial value. For $\psi=1.5$, the tangential thermal conductivity is higher than the radial conductivity. The temperature distribution at the middle of the air-gap, the middle of the PM and the middle of the stator slot for different values of $\psi$ is shown in Figs. $31 \sim 33$. It can be observed that the heat transfer in the machine is better when the tangential thermal conductivity of materials is higher than the radial conductivity. In this case, the temperature is lower. This remark is valid for the studied case where all materials of the machine have the same anisotropy coefficient, which is not true. A more realistic study should take into consideration consider the real values of thermal conductivity anisotropy in each region (i.e., slots, stator and rotor iron cores, PM). Moreover, it is important to note that the conductivity in the $z$-direction of rotating electrical machines is mostly affected by materials anisotropy and a 3-D study is appropriate.

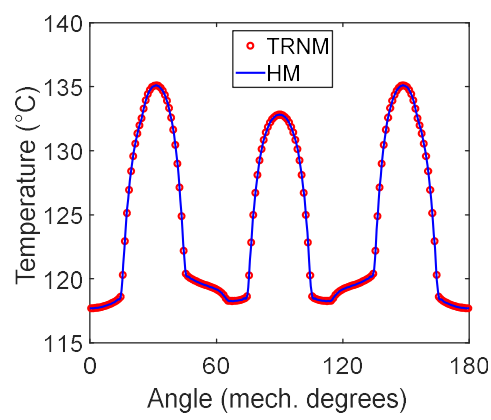

(a)

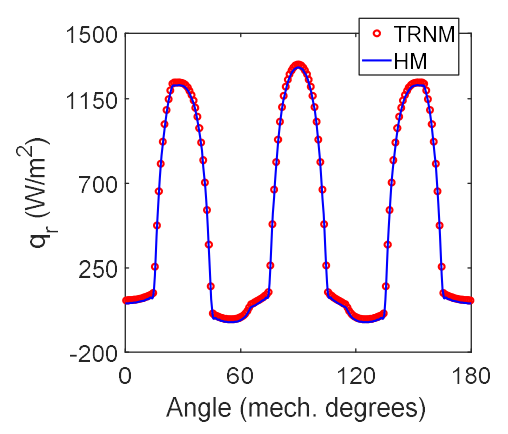

(b)

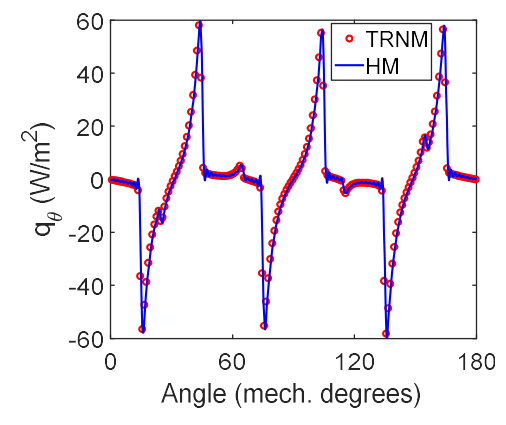

(c)

Fig. 30: Temperature and heat flux components distribution in the middle of the air-gap.

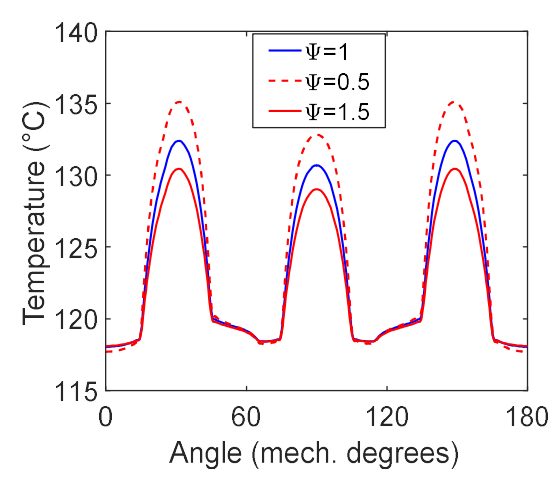

Fig. 31: Temperature distribution in the middle of the air-gap using HM.

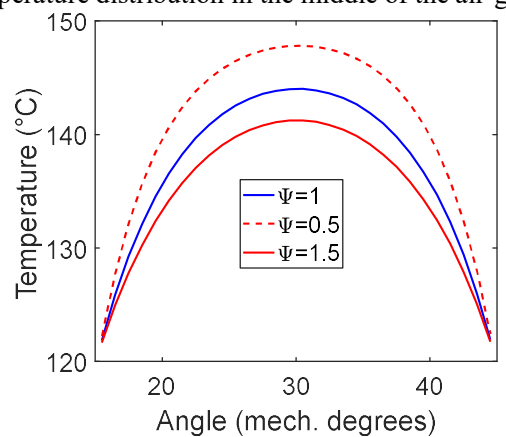

(a)

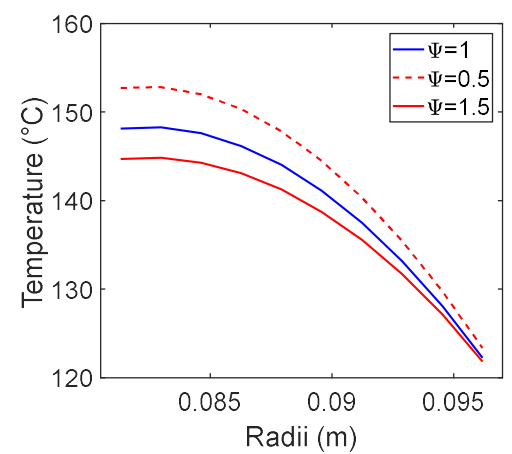

(b)

Fig. 32: Temperature in the middle of the first stator slot using HM.

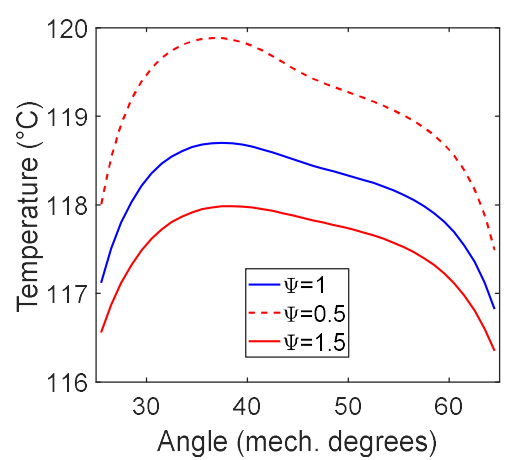

(a) 


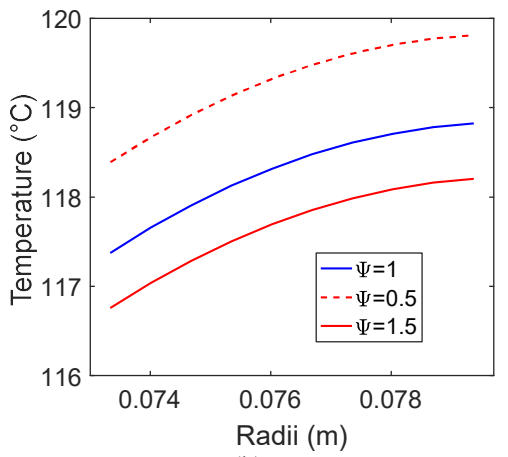

(b)

Fig. 33: Temperature in the middle of the first PM.

\section{CONCLUSION}

The prediction of heat transfer in rotating electrical machines is usually performed using TEC and Fem. Recently, AM and TRNM have been introduced. In this paper, we have proposed an improved 2-D AM based on the exact SD technique, TRNM and $\mathrm{HM}$ for the prediction of steady-state temperature and heat flux components in an inset-PM machine with the materials anisotropy of thermal conductivity. The models are valid for most rotating electrical machines and allow the heat transfer in the electrical machine to be determined with very good accuracy. In the AM, the EDPs representing heat transfer in the electrical machine were solved using the separation of variables method with the thermal conductivity anisotropy in both directions (viz., $r$ and $\theta$ ). The implementation of TRNM in terms of mesh size, connectivity matrix, global matrix and introduction of BCs is presented where its equivalence with Fem is highlighted. As in Fem, the accuracy of TRNM depends on the adopted mesh and the number of elements.

For rotating electrical machines, AM can exhibit lower accuracy when the number of stator slots and poles is important. In this case, the number of ICs in the $r$ - and $\theta$-direction is important; the dimensions of SDs and the harmonics number are small. TRNM is an alternative to AM in these regions with small dimensions and the other regions can be modeled using AM. The coupling between the two methods in HM based on the discrete Fourier series is presented and validated with Fem and TRNM. All results obtained by AM, TRNM and HM are in good agreement with each other and with those obtained by Fem.

\section{APPENDIX A}

We start with the internal elements of the conductive problem example as:

For $k$ from 1 to 32 do

$$
\begin{aligned}
\operatorname{gm}(k, i s k s(1, k)) & =-\frac{1}{R_{k \theta}+R_{i s k s(1, k) \theta}} ; \\
\operatorname{gm}(k, i s k s(3, k)) & =-\frac{1}{R_{k \theta}+R_{i s k s(3, k) \theta}} ; \\
\operatorname{gm}(k, i s k s(4, k)) & =-\frac{1}{R_{k r}+R_{i s k s(4, k) r}} ; \\
\operatorname{gm}(k, i s k s(5, k)) & =-\frac{1}{R_{k r}+R_{i s k s}(5, k) r} ; \\
\operatorname{gm}(k, i s k s(2, k)) & =-(\operatorname{gm}(k, i s k s(1, k))+\operatorname{gm}(k, i s k s(3, k)) . . \\
. .+ & \operatorname{gm}(k, i s k s(4, k))+\operatorname{gm}(k, i s k s(5, k))) ;
\end{aligned}
$$

end do
The equations of thermal convection at the rotor shaft are assembled in the global matrix $\mathrm{gm}$ by

For $k$ from 33 to 40 do

$$
\begin{aligned}
& \operatorname{gm}(k, i s k s(5, k))=-\frac{1}{R_{k r}+R_{i s k s}(5, k) r} ; \\
& g m(k, i s k s(2, k))=-g m(k, i s k s(5, k)) ; \\
& \text { end do }
\end{aligned}
$$

The equations of thermal convection at the ambient air are assembled in the global matrix gm by

For $k$ from 41 to 48 do

$$
\operatorname{gm}(k, i s k s(4, k))=-\frac{1}{R_{k r}+R_{i s k s(4, k) r}} ;
$$

$g m(k, i s k s(2, k))=-g m(k, i s k s(4, k)) ;$

end do

The fixed temperature of $70{ }^{\circ} \mathrm{C}$ is introduced in the global matrix as in Fem by

For $k$ from 33 to 48 do

$g m(k, k)=g m(k, k)+10 E 30$;

$f(k)=10 E 30 .(70+273.16)$;

end do

\section{APPENDIX B}

We start with the nodes of TRNM representing the rotor slots and teeth as

\section{For $k$ from 1 to nsgr do}

$$
\begin{aligned}
& \text { If } k \in \text { Bdrti then } \\
& \operatorname{gm}(k, i s k s(1, k))=-\frac{1}{R_{k \theta}+R_{i s k s(1, k) \theta}} ; \\
& \operatorname{gm}(k, i s k s(3, k))=-\frac{1}{R_{k \theta}+R_{i s k s(3, k) \theta}} ; \\
& \operatorname{gm}(k, i s k s(4, k))=-\frac{1}{R_{k r}} \\
& \operatorname{gm}(k, i \operatorname{sks}(5, k))=-\frac{1}{R_{k r}+R_{i s k s(5, k) r}} ; \\
& g m(k, i s k s(2, k))=-(\operatorname{gm}(k, i s k s(1, k))+\operatorname{gm}(k, i s k s(3, k)) . \\
& . .+\operatorname{gm}(k, i s k s(4, k))+\operatorname{gm}(k, i s k s(5, k))) \text {; }
\end{aligned}
$$

elif $k \in$ Bdrto then

$$
\begin{gathered}
g m(k, i s k s(1, k))=-\frac{1}{R_{k \theta}+R_{i s k s(1, k) \theta}} ; \\
g m(k, i s k s(3, k))=-\frac{1}{R_{k \theta}+R_{i s k s}(3, k) \theta} ; \\
g m(k, i s k s(5, k))=-\frac{1}{R_{k r}} ; \\
g m(k, i s k s(4, k))=-\frac{1}{R_{k r}+R_{i s k s(4, k) r}} ; \\
\operatorname{gm}(k, i s k s(2, k))=-(\operatorname{gm}(k, i s k s(1, k))+\operatorname{gm}(k, i s k s(3, k)) . .
\end{gathered}
$$

elif ( $k \notin B d r t o)$ and $(k \notin B d r t i)$ then

$$
\operatorname{gm}(k, i s k s(1, k))=-\frac{1}{R_{k \theta}+R_{i s k s(1, k) \theta}} ;
$$




$$
\begin{gathered}
g m(k, i s k s(3, k))=-\frac{1}{R_{k \theta}+R_{i s k s}(3, k) \theta} ; \\
g m(k, i s k s(4, k))=-\frac{1}{R_{k r}+R_{i s k s}(4, k) r} ; \\
g m(k, i s k s(5, k))=-\frac{1}{R_{k r}+R_{i s k s}(5, k) r} ; \\
g m(k, i s k s(2, k))=-(\operatorname{gm}(k, i s k s(1, k))+\operatorname{gm}(k, i \operatorname{sis}(3, k)) . . \\
. .+\operatorname{gm}(k, i s k s(4, k))+\operatorname{gm}(k, i s k s(5, k))) ; \\
\text { end if }
\end{gathered}
$$

The introduction of equations (62) to (64) in the global matrix gm is done as follow. (62) is introduced by

$$
\begin{aligned}
& g m(n s g b+1, n s g b+6+9 n n)=-1 \\
& g m(n s g b+1, n s g b+5+8 n n)=-\ln \left(R_{r}\right) \\
& \text { For } j \text { from } 1 \text { to } n s d o \\
& \quad k=n o d b(1,1, j) \\
& \quad \operatorname{gm}(n s g b+1, k)=1 /(2 . n n)
\end{aligned}
$$

end do

$f(n s g b+1)=-\frac{1}{4} \frac{q_{r} R_{r}^{2}}{\lambda_{r r}}$

(63) gives

For $n$ from 1 to nn do

$$
\begin{aligned}
& \operatorname{gm}(n s g b+1+n, n s g b+6+9 . n n+n)=-\left(\frac{R_{r}}{R_{i}}\right)^{-n \cdot \tau_{r}} \\
& \operatorname{gm}(n s g b+1+n, n s g b+5+8 . n n+n)=-1 \\
& \text { For j from } 1 \text { to } n s d o \\
& \quad \operatorname{k}=n o d b(1,1, j) \\
& \quad g m(n s g b+1+n, k)=\frac{\cos \left(n \cdot \theta_{k}\right)}{n n}
\end{aligned}
$$

end do

end do

(64) is added to gm as

For $n$ from 1 to $n$ n do

$$
\begin{aligned}
& g m(n s g b+1+n n+n, n s g b+6+11 . n n+n)=-\left(\frac{R_{r}}{R_{i}}\right)^{-n \cdot \tau_{r}} \\
& g m(n s g b+1+n n+n, n s g b+6+10 . n n+n)=-1
\end{aligned}
$$

For jrom 1 to $n s$ do

$$
\begin{aligned}
& \mathrm{k}=\operatorname{nodb}(1,1, \mathrm{j}) \\
& g m(n s g b+1+n n+n, k)=\frac{\sin \left(n \cdot \theta_{k}\right)}{n n}
\end{aligned}
$$

end do

end do

There are 360 equations (66) to be introduced in the global matrix depending on the number of boundary nodes at $R_{r}$. They are given as

For j from 1 to ns do

$$
\begin{aligned}
& k=\operatorname{nodb}(1,1, j) \\
& \operatorname{gm}(k, i s k s(5, k))=\frac{1}{R_{i s k s(5, k) r}} \\
& \operatorname{gm}(k, i s k s(2, k))=-\operatorname{gm}(k, i s k s(5, k)) \\
& \operatorname{gm}(k, n s g b+5+8 . n n)=-L_{u} \lambda_{r r}(2 . d \theta)
\end{aligned}
$$

$$
f(k)=-\frac{1}{2} R_{r}^{2} L_{u} q_{r}(2 . d \theta)
$$

For $n$ from 1 to $n$ n do

$$
\begin{aligned}
& g m(k, n s g b+5+8 . n n+n)= \\
& -L_{u} \lambda_{r r} \tau_{r}\left(\sin \left(n \cdot\left(d \theta-\theta_{k}\right)\right)+\sin \left(n \cdot\left(d \theta+\theta_{k}\right)\right)\right) \\
& g m(k, n s g b+6+9 . n n+n)= \\
& L_{u} \lambda_{r r} \tau_{r}\left(\frac{R_{r}}{R_{i}}\right)^{-n \cdot \tau_{r}}\left(\sin \left(n \cdot\left(d \theta-\theta_{k}\right)\right)+\sin \left(n \cdot\left(d \theta+\theta_{k}\right)\right)\right) \\
& g m(k, n s g b+6+10 . n n+n)= \\
& -L_{u} \lambda_{r r} \tau_{r}\left(\cos \left(n \cdot\left(d \theta-\theta_{k}\right)\right)-\cos \left(n \cdot\left(d \theta+\theta_{k}\right)\right)\right) \\
& g m(k, n s g b+6+11 . n n+n)= \\
& L_{u} \lambda_{r r} \tau_{r}\left(\frac{R_{r}}{R_{i}}\right)^{-n \cdot \tau_{r}}\left(\cos \left(n \cdot\left(d \theta-\theta_{k}\right)\right)-\cos \left(n \cdot\left(d \theta+\theta_{k}\right)\right)\right)
\end{aligned}
$$

end do

end do

\section{ACKNOWLEDGMENT}

The authors acknowledge the financial support of the General Directorate of Scientific Research and Technological Development (DGRSDT) of Algeria.

\section{REFERENCES}

[1] L. Siesing, A. Reinap, and M. Andersson, "Thermal Properties on High fill factor electrical windings: Infiltrated vs non infiltrated", International Conference on Electrical Machines (ICEM), pp. 22182223, 2014. https://doi.org/10.1109/ICELMACH.2014.6960492).

[2] C.-C. Hwang, S.S. Wu, and Y.H. Jiang, "Novel Approach to the Solution of Temperature Distribution in the Stator of an Induction Motor”, IEEE Trans. Energy Conv., vol. 15, no. 4, pp. 401-406, Dec. 2000. https://doi.org/10.1109/60.900500

[3] X. Cai, M. Cheng, S. Zhu, and J. Zhang, "Thermal Modeling of FluxSwitching Permanent-Magnet Machines Considering Anisotropic Conductivity and Thermal Contact Resistance", IEEE Trans. Ind. Elect., vol. 63, no. 6, pp. 3355-3365, Jun. 2016. https://doi.org/ 10.1109/TIE.2016.2522942

[4] Q. Chen, D. Liang, L. Gao, Q. Wang, and Y. Liu, "Hierarchical thermal network analysis of axial-flux permanent-magnet synchronous machine for electric motorcycle", IET Electric Power Applications, vol. 12, no. 12, pp. 859-866, 2018. https://doi.org/10.1049/iet-epa.2017.0719

[5] Y. Yang, B. Bilgin, M. Kasprzak, S. Nalakath, H. Sadek, M. Preindl, J. Cotton, N. Schofield, and A. Emadi, "Thermal Management of Electric Machines", IET Electrical Systems in Transportation, vol. 7, no. 2, pp. 104-116, 2017. https://doi.org/10.1049/iet-est.2015.0050

[6] R. Wrobel, P. Mellor, and D. Holliday, "Thermal Analysis of a Segmented Stator Winding Design", IEEE Trans. Ind. Appl., vol. 47, no. 5, pp. 2023-2030, Sep./Oct. 2011. https://doi.org/10.1109/ECCE.2010.5617814

[7] K. Boughrara, F. Dubas, and R. Ibtiouen, "2-D Exact Analytical Method for Steady-State Heat Transfer Prediction in Rotating Electrical Machines", IEEE Trans. Magn., vol. 54, no. 9, pp. 1-19, Sep. 2018, Art. ID 8104519. https://doi.org/10.1109/TMAG.2018.2851212

[8] F. Dubas, and K. Boughrara, "New scientific contribution on the 2-D subdomain technique in Cartesian coordinates: Taking into account of iron parts," Math. Comput. Appl., vol. 22, no. 1, p. 17, Feb. 2017. https://doi.org/ 10.3390/mca22010017

[9] F. Dubas, and K. Boughrara, "New scientific contribution on the 2-D subdomain technique in polar coordinates: Taking into account of iron parts," Math. Comput. Appl., vol. 22, no. 4, p. 42, Oct. 2017 https://doi.org/ 10.3390/mca22040042

[10] H. Ennassiri, G. Barakat, and Y. Amara, "Steady state hybrid thermal modeling of permanent magnet electrical machines," International Conference on Ecological Vehicles and Renewable Energies (EVER), pp. 1-6, 2016. https://doi.org/10.1109/EVER.2016.7476420.

[11] S. Ouaged, M.A. Ben Hamida, Y. Amara, G. Barakat, and J.J.H. Paulides, "Thermal modelling of Tubular Linear Machines using a Hybrid Analytical Method," International Conference on Sustainable Mobility Applications, Renewables and Technology (SMART), pp. 1-5, 2015. https://doi.org/10.1109/SMART.2015.7399242

[12] D. Jackson, "On the Order of Magnitude of the Coefficients in Trigonometric Interpolation", Transactions of the American 
Mathematical Society, 21, 1920, pp. 321-332, https://doi.org/10.1090/S0002-9947-1920-1501147-2

[13] D.C. Meeker, "Rotating Losses in a Surface Mount Permanent Magnet Motor", October 2017, http://www.femm.info/wiki/SPMLoss.

[14] K-H. Shin, K. Hong, H-W. Cho, and J-Y. Choi, "Core Loss Calculation of Permanent Magnet Machines Using Analytical Method", IEEE Trans. on Applied Superconductivity, vol. 28, no. 3, pp. 1-5, April 2018, Art. ID 5205005. https://doi.org/10.1109/TASC.2018.2800706

[15] Sh. Utegenova, F. Dubas, M. Jamot, R. Glises, B. Truffart, D. Mariotto, $\mathrm{P}$. Lagonotte, and Ph. Desevaux, "An Investigation Into the Coupling of Magnetic and Thermal Analysis for a Wound-Rotor Synchronous Machine," IEEE Trans. Ind. Elect., vol. 65, no. 4, pp. 3406-3416, Apr. 2018. https://doi.org/10.1109/TIE.2017.2756597

[16] Z. Djelloul-khedda, K. Boughrara, F. Dubas, A. Kechroud, and A. Tikellaline, "Analytical Prediction of Iron Core Losses in FluxModulated Permanent-Magnet Synchronous Machines", IEEE Trans. Magn., 2018. https://doi.org/ 10.1109/TMAG.2018.2877164

[17] D.C. Meeker. (Apr. 1, 2009). Finite Element Method Magnetics ver. 4.2. [Online]. Available: http://www.femm.info.

[18] E. Divo and A. Kassab, "On a Boundary-Only BEM for Heat Conduction in Non-Homogeneous Anisotropic Media", Transactions on Modeling and Simulation, vol. 15, pp. 279-288, WIT Press,1997.

[19] Flux2D, General Operating Instructions, Version 2017; Altair.

[20] G. Barakat, and Y. Amara, "A simple and effective way to couple analytical formal solution of magnetic potential and reluctance network models", International Conference on Computation in Electromagnetics (CEM), pp. 1-2, 2014, https://doi.org/10.1049/cp.2014.0199

[21] K.J.W. Pluk, J.W. Jansen, and A. Lomonova, "Hybrid Analytical Modeling: Fourier Modeling Combined With Mesh-Based Magnetic Equivalent Circuits", IEEE Trans. Magn., vol. 51, no. 8, pp. 1-12, Aug. 2015, Art. ID 8106812. https://doi.org/10.1109/TMAG.2015.2419197

[22] M. Johnson, M.C. Gardner, and H.A. Toliyat, "A Parameterized Linear Magnetic Equivalent Circuit for Analysis and Design of Radial Flux Magnetic Gears-Part I: Implementation", IEEE Trans. Energy Conv., vol. 33, no. 2, pp. 784-791, June 2018. https://doi.org/10.1109/TEC.2017.2777875

[23] M. Johnson, M.C. Gardner, and H.A. Toliyat, "A Parameterized Linear Magnetic Equivalent Circuit for Analysis and Design of Radial Flux Magnetic Gears-Part II: Evaluation", IEEE Trans. Energy Conv., vol. 33, no. 2, pp. 792-800, June 2018. https://doi.org/10.1109/TEC.2017.2777876

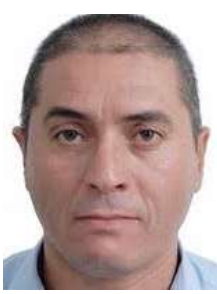

Kamel Boughrara was born in Algiers, Algeria, in 1969. He received the Engineer Diploma degree from Ecole Nationale Polytechnique (ENP), Algiers, in 1994, the Magister degree from the University of Sciences and Technology Houari Boumediene, Algiers, in 1997, and the Ph.D. degree from ENP, in 2008. He is currently a Professor with ENP, where he is also the head of department of Electrical Engineering.

His current research interests include the modeling and control of electrical machines.

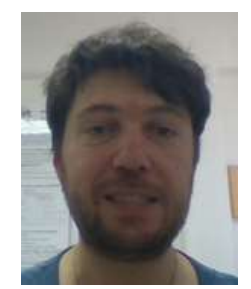

Frédéric Dubas was born in Vesoul, France, in 1978. He received the M.Sc. degree and the Ph.D. degree from the "Univ. Bourgogne Franche-Comté" (Besançon, France) in 2002 and 2006, respectively, with a focus on the design and the optimization of high-speed surface-mounted permanent-magnet (PM) synchronous motor for the drive of a fuel cell air-compressor. From 2014 to 2016, he has been the Head of "Unconventional Thermal and Electrical Machines" Team. He is the Head of the "Electrical Actuators" group in the "Hybrid \& Fuel Cell Systems, Electrical Machines (SHARPAC)" Team. He works with ALSTOM Transports (Ornans, France), and RENAULT Technocenter (Guyancourt, France), where he is involved in the modelling, design and optimization of electrical systems and, in particular, induction and PM synchronous (radial and/or axial flux) machines, creative problem solving, and electrical propulsion/traction. He is currently an Associate Professor with the Dép. ENERGIE, FEMTO-ST Institute affiliated to the CNRS and jointly with the "Univ. Bourgogne Franche-Comté" (Besançon, France).

He has authored over 100 refereed publications and he holds a patent about the manufacturing of axial-flux PM machines with flux-focusing. Dr. Dubas received: i) the Prize Paper Awards in the IEEE Conference Vehicle Power and Propulsion (VPPC) in 2005, ii) the Prize Presentation Awards in the 19th International Conference on Electrical Machines and Systems (ICEMS) in 2017, iii) the RENAULT Internal Award (Direction Engineering Alliance - Innovation) in 2019. 